\title{
Volksentscheide nach dem Sankt-Florians-Prinzip? Das Abstimmungsverhalten zu Stuttgart 21 und großen Infra- strukturprojekten in der Schweiz im Vergleich
}

\author{
Adrian Vatter/Anja Heidelberger
}

\section{Abstract}

Der Beitrag untersucht das Stimmverhalten zu Stuttgart 21 und vergleicht es mit demjenigen bei Volksentscheiden zu grossen Infrastrukturprojekten in der Schweiz. Im Mittelpunkt steht die empirische Überprüfung von Hypothesen, die vom sog. NIMBY-Verhalten (Not in My Back Yard) der Stimmbürger ausgehen. Anhand der Analyse der Volksabstimmung zu Stuttgart 21 und 13 nationalen Abstimmungen zu grossen Bahn-, Autobahn- und AKWProjekten aus der Schweiz bestätigt sich die Relevanz der örtlichen Betroffenheit zur Erklärung des Stimmentscheids. Im Weiteren zeigt sich, dass bei diesen Vorlagen auch die parteipolitische Polarisierung bedeutsam ist und das Muster des Stimmverhaltens der Baden-Württemberger bei Stuttgart 21 nicht demjenigen bei großen Bahnprojekten in der Schweiz entspricht.

\section{Einleitung}

Am 16. Dezember 2010 wählte die Gesellschaft für deutsche Sprache die Begriffe „Wutbürger“ und „Stuttgart 21“ im Wettbewerb um das „Wort des Jahres“ auf die Plätze eins und zwei. Die beiden Begriffe haben nicht nur die öffentliche Debatte der letzten Jahre wesentlich mitbestimmt, sondern auch auf ein offensichtliches Defizit der deutschen Politik aufmerksam gemacht. Sie dokumentieren anhand eines konkreten Beispiels das große Bedürfnis der Bürgerinnen und Bürger, dass ihnen über ihre Wahlentscheidung hinaus ein unmittelbares Mitspracherecht bei gesellschaftlich und politisch relevanten Projekten erteilt werden soll. Entsprechend wird heute der Begriff „Stuttgart $21^{\prime \prime}$ nicht nur mit der Umwandlung des Stuttgarter Kopfbahnhofs in einen 
unterirdischen Durchgangsbahnhof in Verbindung gebracht, sondern auch mit Demonstrationen und Bürgerprotesten, langwierigen politischen Debatten und Entscheiden, aber auch mit Forderungen und Diskussionen um mehr Mitsprache der Bürger und eine direktere Form der Demokratie. Am 27. November 2011 fand die Auseinandersetzung um Stuttgart 21 in einer landesweiten Volksabstimmung über ein Gesetz zur Kündigung der vertraglichen Finanzierungsvereinbarungen ihren bisherigen Höhepunkt und ihr vorläufiges Ende.

Sind Volksentscheide in Deutschland auf Landesebene schon nicht alltäglich, so war hier zudem außergewöhnlich, dass das gesamte Land BadenWürttemberg zur Abstimmung über die Finanzierung eines Projektes in der Stadt Stuttgart aufgerufen wurde. Damit musste die Bürgerschaft über eine Vorlage abstimmen, die je nach Wohnort für den Einzelnen mit ganz unterschiedlichen Lasten und Nutzen verbunden ist. Während das gesamte Bundesland einen beträchtlichen Teil der Kosten trägt, profitieren einzelne Regionen durch eine bessere Erschließung mit dem öffentlichen Verkehr und erhalten damit einen unmittelbaren Nutzen durch das Projekt. Andere Landesteile müssen jedoch befürchten, dass die Finanzmittel des Landes in Milliardenhöhe, die für Stuttgart 21 aufgewendet werden, in näherer Zukunft nicht mehr für weitere lokale Bahnprojekte zur Verfügung stehen werden. In positiver und negativer Hinsicht am stärksten betroffen sind schließlich die Stuttgarter: Einerseits muss die Stadt Stuttgart einen Teil der Kosten übernehmen. Zudem sind die Stuttgarter im Stadtzentrum und beim Verlauf der neuen Bahnlinie direkt dem jahrelangen Baulärm ausgesetzt. Hinzu kommen der von vielen Stuttgartern bekämpfte Abriss eines Teils des denkmalgeschützten Bahnhofgebäudes und der Eingriff in den Stuttgarter Schlosspark. Andererseits sind es auch die Stuttgarter, die am stärksten vom Projekt S21 profitieren. Sie erhalten eine moderne Bahnhofsinfrastruktur, ein attraktives Stadtviertel, größere Grünflächen, raschere Bahnverbindungen sowie zahlreiche neue Arbeitsplätze (Bahnprojekt Stuttgart-Ulm 2012).

Am 27. November 2011 wurde das Gesetz zur Aufkündigung der Finanzierung von Stuttgart 21 mit 58,9\% Nein-Stimmen abgelehnt ${ }^{1}$. Noch am Abstimmungssonntag gestand Baden-Württembergs Ministerpräsident Winfried Kretschmann (Bündnis 90/Grüne) die Niederlage der Stuttgart-21-Gegner ein und sicherte zu, dass nun das Baurecht der Deutschen Bahn akzeptiert würde.

1 Selbstredend kam damit die baden-württembergische Verfassungsregelung, dass mindestens ein Drittel der Stimmberechtigten dem Gesetz zustimmen muss, nicht zur Anwendung. 
Eine vorgängig durchgeführte Umfrage zeigte zudem, dass über $90 \%$ der Bevölkerung Baden-Württembergs das Ergebnis des Bürgerverdikts unabhängig vom Ausgang akzeptieren würden ${ }^{2}$. Die Volksabstimmung zum jahrelangen und teilweise heftig ausgetragenen Konflikt über den Bau des Bahnprojekts Stuttgart 21 scheint damit zu einer nachhaltigen Entspannung der politisch verfahrenen Situation geführt zu haben.

Die Volksabstimmung zu Stuttgart 21 wird kein Einzelfall bleiben. Lokaler Widerstand gegen große Infrastrukturprojekte macht sich vielerorts breit, und beinahe täglich wird in den Medien über Bürgerproteste gegen Flugpisten, Atomendlager oder Hochspannungsleitungen berichtet. In Zukunft wird man deshalb vermehrt die Lösung im direktdemokratisch legitimierten Entscheid der Bürger suchen, wenn klassische Planfeststellungsverfahren, nachträgliche Mediationen und staatlicher Zwang am Widerstand der betroffenen Bürgerschaft scheitern. Allerdings wissen wir bis heute wenig darüber, welche Faktoren das Stimmverhalten der Bürgerinnen und Bürger bei grossen Infrastrukturprojekten beeinflussen. Fundierte Kenntnisse über die politische Akzeptanz der Bevölkerung zu konkreten Infrastrukturmassnahmen sind aber eine notwendige Voraussetzung für die erfolgreiche Gestaltung einer zukünftigen Infrastrukturpolitik und der adäquaten Bereitstellung öffentlicher Güter durch den Staat. Die Analyse von Daten aus Abstimmungen bildet damit eine wertvolle Grundlage, um Informationen über den perzipierten Nutzen und die Akzeptanz öffentlicher Leistungsangebote und ihrer Standortwahl durch die Bevölkerung zu erfassen.

Der vorliegende Artikel soll einen Beitrag dazu leisten und folgende Fragen beantworten: Welche Rolle spielen die lokale Betroffenheit und damit die räumliche Nähe für die Erklärung des Stimmbürgerverhaltens bei Volksentscheiden zu großtechnischen Infrastrukturprojekten mit Nutzenstreuungen? Stimmen die Bürger großen Infrastrukturvorhaben nur zu, wenn nicht vor der eigenen Haustüre gebaut wird? Welche weiteren Bestimmungsgrößen sind relevant für die Analyse von Volksabstimmungen zu öffentlichen Infrastrukturvorhaben? Den Untersuchungsgegenstand der vorliegenden Studie bildet zunächst die Volksabstimmung über das Bahnprojekt Stuttgart 21. Im Weiteren werden die zahlreichen Volksentscheide in der Schweiz zu großen Infrastrukturprojekten von nationaler Bedeutung mit ungleicher räumli-

2 Siehe hierzu etwa die Umfrage „Stimmung vor dem Volksentscheid zu Stuttgart 21“ von Infratest dimap bei 2403 Befragten im November 2011 (Bertelsmann Stiftung und Universität Stuttgart) sowie auch die Umfragestudie von Faas u. Schmitt-Beck (2012). 
cher Verteilung konkreter Lasten und Nutzen analysiert, wobei zwischen den drei relevanten Themenfeldern „Eisenbahnbau“, „Autobahnbau“ und „Atomkraftwerkbau“ unterschieden wird.

Die Studie geht in zweierlei Hinsicht über den bisherigen Forschungsstand hinaus: (1) Theoretisch versucht sie zum ersten Mal, eine systematische quantitative Überprüfung des sogenannten NIMBY-Phänomens ${ }^{3}$ anhand der effektiven Abstimmungsergebnisse bei Volksentscheiden zu großtechnischen Infrastrukturprojekten mit Nutzenstreuungen vorzunehmen. Zwar bestehen zahlreiche qualitative Fallstudien und Umfragen aus dem Bereich der PolicyForschung, die sich mit dem NIMBY-Phänomen anhand von Mülldeponien, Atomkraftwerken und -endlagern sowie Hochspannungsleitungen auseinandergesetzt haben (u.a. Dear 1980; Frey 1997; Frey u.a. 1996; Van der Horst 2007; Warren 2005). Bis heute wurden aber das tatsächliche Stimmbürgerverhalten bei entsprechenden Volksentscheiden kaum untersucht und die einander befruchtenden Erkenntnisse der Policy-Analyse und der politischen Einstellungsforschung nicht miteinander verknüpft. (2) Empirisch handelt sich um einen der ersten Beiträge zum Stimmverhalten bei einer Volksabstimmung in Deutschland ${ }^{4}$ sowie die erste Studie, die gleichzeitig das Stimmbürgerverhalten auf der Basis räumlicher Daten von deutschen und schweizerischen Volksentscheiden untersucht. Aufgrund der ähnlichen Größenverhältnisse der Schweiz und Baden-Württembergs in Bezug auf Bevölkerungsgröße (CH: 7,9 Mio; BW: 10,8 Mio), Fläche (CH: $41.285 \mathrm{~km}^{2}$; BW: $35.751 \mathrm{~km}^{2}$ ) und wirtschaftliche Leistungskraft ${ }^{5}$, ihrer räumlichen und kulturellen Nähe sowie ähnlicher Problemlagen bei der Bereitstellung großtechnischer Infrastrukturprojekte erscheint ein Vergleich der baden-württembergischen S21-Volksabstimmung mit Volksentscheiden zu Infrastrukturvorhaben aus der Schweiz besonders aussagekräftig.

3 NIMBY steht als englisches Akronym für Not In My Back Yard (Deutsch: Nicht in meinem Hinterhof). Eine andere dafür auch häufige Bezeichnung ist das sog. LULU-Phänomen (für Locally Unwanted Land Uses). Der gängige deutsche Ausdruck ist das sogenannte Sankt-Florians-Prinzip (bzw. Sankt-Florians-Politik): Heiliger Sankt Florian, verschon ' mein Haus, zünd' andre an!

4 Die wenigen Ausnahmen stellen die aufschlussreichen Analysen von Glantz u.a. (2011) und Schoen (2012) zum Stimmverhalten beim Volksentscheid zum Nichtraucherschutz in Bayern von 2010 dar. In diesem Zusammenhang aussagekräftig sind auch die Studien von Eder (2010a, b) über die Erfolgsfaktoren deutscher Volksentscheide.

5 BIP pro Einwohner (CH: 49.700 Euro; BW: 33.651 Euro); Arbeitslosenquote (CH: $3,3 \%$; BW: $3,7 \%$ ). 
Der Artikel ist wie folgt aufgebaut: Im nächsten Abschnitt werden die Leithypothesen zum NIMBY-Phänomen hergeleitet und weitere Kontrollhypothesen vorgestellt. Im dritten Abschnitt werden die Untersuchungsanlage und die Operationalisierung der Variablen präsentiert. Darauf folgen im vierten Abschnitt die empirischen Ergebnisse, bevor wir im letzten Abschnitt Folgerungen ziehen.

\section{Theorien und Hypothesen}

In der Abstimmungsforschung werden vor allem soziokulturelle, soziostrukturelle und parteipolitische Erklärungsstränge sowie der Rational ChoiceAnsatz beigezogen, um das Verhalten der Stimmbürgerinnen und Stimmbürger bei Sachvorlagen zu erklären (Bowler/Donovan 1998; Buri/Schneider 1993; Freitag/Wagschal 2007; Kriesi 2005; LeDuc 2002; Wagschal 2007) ${ }^{6}$. Bei Abstimmungen in der Schweiz spielen entsprechend auch parteipolitische, soziologische oder kulturelle Faktoren eine wichtige Rolle (Bühlmann u.a. 2006; Milic 2008; Sciarini/Tresch 2009; Vatter u.a. 2000; Vatter/Nabholz 1995). Der Stimmentscheid bei großtechnischen Infrastrukturvorhaben mit Spillovers, so unsere Leithypothese, beruht aber in erster Linie auf einem Kosten-Nutzenkalkül, bei der die negative lokale Betroffenheit, also die räumliche Distanz zum Abstimmungsgegenstand, von herausragender Bedeutung ist. Dieses sog. NIMBY-Phänomen stellt dabei eine Variante des „Rational Choice“-Ansatzes dar. So geht die Theorie der Neuen Politischen Ökonomie davon aus, dass sich der rationale Bürger unter einer Menge verfügbarer Alternativen für diejenige entscheidet, die seinen erwarteten Nutzen maximiert (Downs 1957; Frey 1997; Vatter 1994). Abstimmen wird hier als ein instrumenteller Akt verstanden, um den eigenen Nutzen zu maximieren, während nicht-instrumentelle Faktoren wie tradierte Wertehaltungen und affektive Parteibindungen keinen Einfluss auf das Stimmverhalten ausüben. Entscheidend ist die perzipierte Kosten-Nutzen-Bilanz der Stimmbürger, die schließlich zum Stimmentscheid führt: Übersteigt der Grenznutzen die Grenzkosten der Stimmenden, wird das Sachgeschäft in einer Volksabstimmung angenommen (Frey 1997). Bei der Analyse von Volksabstim-

6 Da im Folgenden die Analyse von Aggregatdaten im Vordergrund steht, wird der für Individualdatenstudien oft verwendete sozialpsychologische Ansatz hier nicht weiter verfolgt, ebenso nicht die Rolle von Kampagneneffekten. Eine vertiefte Analyse des individuellen Meinungsbildungsprozesses im Vorfeld der Volksabstimmung zu Stuttgart 21 findet sich bei Faas/Schmitt-Beck (2012). 
mungen, bei denen die Sachvorlage offensichtliche räumliche Spillovers auslöst, verwenden Vertreter des polit-ökonomischen Ansatzes deshalb regelmäßig die geographische Distanz zum Abstimmungsgegenstand als einen Indikator, um das Nutzenkalkül der Stimmbürger zu erfassen und daraus auf die Nachfrage nach öffentlichen Gütern und Dienstleistungen zu schließen (Greenberg/Amer 1989; Schneider/Pommerehne 1985; Blöchliger/Spillmann 1992). In diesem Sinne lassen sich die im Folgenden hergeleiteten NIMBYHypothesen dem erweiterten „Rational Choice“-Ansatz zur Erklärung des Stimmbürgerverhaltens zuordnen ${ }^{7}$, wobei ihr Vorteil gegenüber der konventionellen Rational Choice-Theorie darin liegt, dass es sich um induktive und empirisch eindeutig überprüfbare Hypothesen handelt.

\section{Leithypothesen zum NIMBY-Stimmbürger}

Der Ausgangspunkt aller Bürgerproteste gegen grosse Infrastrukturprojekte ist das Auseinanderfallen von Lasten und Nutzen. Während die Anwohner einer grosstechnischen Anlage jahrelangen Baulärm, erhöhte Umwelt- und Gesundheitsrisiken, sinkende Grundstückspreise oder ein beeinträchtigtes Landschaftsbild ertragen müssen, profitiert der übrige Teil der Bevölkerung vom gesamtgesellschaftlichen Nutzen dieser öffentlichen Güter. Typischerweise weisen die direkten negativen Auswirkungen dieser Großprojekte häufig nur eine räumlich begrenzte Reichweite auf, wodurch sich für die Standortregion eine andere Kosten-Nutzen-Bilanz ergibt als für weiter entfernte Regionen. Damit liegen bei der Realisierung solcher Infrastrukturvorhaben Bedingungen vor, die je nach Betroffenheitsgrad ein ganz unterschiedliches politisches Verhalten der Bevölkerung erwarten lassen. Eine in diesen Fällen oft auftretende Reaktionsweise wird in der Literatur als sogenanntes NIMBY-Phänomen oder Sankt-Florians-Prinzip beschrieben (Dear 1992; Kraft 1991; Rabe 1994; Wolsink 1994). Es steht für eine politische Grundhaltung, die sich dadurch auszeichnet, dass die Bürger einerseits öffentliche Infrastrukturleistungen mit positiven Nutzen für die Allgemeinheit befürworten, gleichzeitig aber mit Vehemenz dagegen opponieren, wenn diese Infrastrukturprojekte mit ihren lokal begrenzten Lasten in ihrer unmittelbarer Nachbarschaft gebaut werden. Entsprechend beschreibt van der Horst (2007,

7 In diesem Sinne auch Schively (2007) sowie Hunter/Leyden (1995: 613): „To a large extent the NIMBY label is grounded in rational choice theory, which assumes that human behavior is based on self-interest, narrowly conceived." 
S. 2705) das typische NIMBY-Verhalten so, ,that certain services are in principle considered as beneficial by the majority of the population, but that proposed facilities to provide these services are in practice often strongly opposed by local residents." Dear (1992) betont dabei verschiedene Faktoren, die für die typische NIMBY-Verhaltensweise der Anwohner charakteristisch sind: Als weitaus wichtigsten Einflussfaktor betrachtet er die räumliche Distanz zum Objekt. Er geht davon aus, dass die Zustimmung zu Infrastrukturanlagen mit zunehmender geographischer Distanz zunimmt: „There is one universal factor in all NIMBY conflicts: geographical proximity. The rule is simple: The closer residents are to an unwanted facility, the more likely they are to oppose it" (Dear 1992, S. 294).

Warren (2005, S. 866) weist allerdings auch auf die Möglichkeit der umgekehrten Situation hin: In seiner Studie über den Effekt von Windkraftwerken sinkt der Widerstand gegen die Projekte mit zunehmender Nähe. Die Anwohner sprechen sich deutlich stärker für das Infrastrukturvorhaben aus als die Allgemeinheit. Warren (2005) bezeichnet diesen Fall, der etwa bei „sauberen“ Energieträgern auftritt, deshalb als ein ,inverse NIMBYsyndrome" oder kurz als YIMBY-Phänomen (Yes In My Back Yard).

Welchem der beiden Phänomene nun eine Vorlage zugeordnet werden kann, ist damit abhängig von der unterschiedlichen Perzeption der KostenNutzen-Rechnung durch die Gesamtbevölkerung und die Direktbetroffenen. Theoretisch ist je nach Infrastrukturprojekt ein positiver oder negativer Einfluss der räumlichen Distanz auf die Zustimmung zu erwarten, weshalb zunächst zwei Hypothesen formuliert werden.

H1a: Stimmbürger von Kommunen, die näher bei den betreffenden Infrastrukturprojekten wohnen, lehnen die Vorlage stärker ab als die übrigen Stimmbürger (NIMBY).

H1b: Stimmbürger von Kommunen, die näher bei den betreffenden Infrastrukturprojekten wohnen, sprechen sich stärker für die Vorlage aus als die übrigen Stimmbürger (YIMBY).

Dear (1980) stellt zudem fest, dass sich die Zustimmung nicht über die gesamte Distanz systematisch verändert, sondern nur in einem begrenzten Bereich. Ab einer gewissen Entfernung sind die Bürger den Projekten gegenüber indifferent. Entsprechend kann auch für die zu untersuchenden Volksentscheide vermutet werden, dass ab einer gewissen räumlichen Entfernung keine distanzabhängigen Faktoren mehr einen Einfluss auf den Stimmentscheid ausüben und sich die Zustimmung daher nicht mehr wesentlich verändert.

H2a bzw. H2b: Die räumliche Distanz übt nur in einem örtlich begrenzten Bereich einen direkten positiven (bzw. negativen) Einfluss auf den Stimmentscheid aus. 
Um bei den Untersuchungsfällen sicherzustellen, dass der Einfluss der Distanz nicht auf spezifische regionale (d.h. kreis- bzw. kantonsspezifische) Unterschiede zurückzuführen ist, sondern effektiv die räumliche Nähe der Kommunen zum Projektstandort entscheidend ist, wird zusätzlich überprüft, ob der Zusammenhang zwischen Distanz und Stimmentscheid auch innerhalb eines Kantons respektive innerhalb eines Kreises zu finden ist.

H3a bzw. H3b: Innerhalb eines Kantons bzw. eines Kreises stimmen die weiter entfernten (bzw. näher gelegenen) Kommunen stärker für Infrastrukturprojekte als die näher gelegenen (bzw. weiter entfernten).

Neben dem materiellen Entscheidverhalten nimmt in der NIMBY-Literatur auch die Mobilisierung der lokal Betroffenen eine prominente Rolle ein. So wird argumentiert, dass die negative Betroffenheit der Anwohner vor allem auch zu einem stärkeren persönlichen Engagement und einer größeren politischen Involvierung gegen große Infrastrukturprojekte in der unmittelbaren Nachbarschaft führt (Dear 1992). Bei Volksabstimmungen drückt sich diese stärkere Mobilisierung der Direktbetroffenen in einer höheren Stimmbeteiligung aus. Trifft die NIMBY-Mobilisierungsthese zu, dann müsste in den direktbetroffenen Gebieten, die näher beim Abstimmungsobjekt liegen, eine deutlich höhere Stimmbeteiligung sichtbar sein als in weiter entfernten Regionen.

H4: Je näher die Stimmbürger bei einem Projektstandort wohnen, desto höher ist die Stimmbeteiligung.

Wenn eine solche Korrelation besteht, könnte die Höhe der Stimmbeteiligung auch den Zusammenhang zwischen Distanz und Stimmentscheid beeinflussen. Wenn - wie wir in Hypothese $2 \mathrm{a}$ und $2 \mathrm{~b}$ vermuten - die Betroffenheit nur auf einen kleinen Kreis begrenzt ist, sollte die Distanz hauptsächlich in diesen direktbetroffenen Gebieten eine Rolle spielen. Es wird deshalb postuliert, dass der Einfluss der Distanz auf den Stimmentscheid besonders groß ist, wenn die Stimmbeteiligung hoch ist.

H5: Je höher die Stimmbeteiligung ist, desto stärker wirkt die Distanz auf den Stimmentscheid.

Ein weiteres Merkmal von Dear (1992) zur Erfassung des NIMBYPhänomens betrifft die jeweilige Charakteristik des Infrastrukturbereichs ${ }^{8}$. Im Grundsatz geht er davon aus, dass je nach Sachbereich ein unterschiedlich großes Potential für lokalen Widerstand sowie eine Akzeptanzhierarchie

8 Dear (1992: 294) beschreibt das NIMBY-Phänomen anhand von Einrichtungen für psychisch Kranke, Alkoholkranke und Obdachlose. 
in Bezug auf die Unerwünschbarkeit von Infrastruktureinrichtungen besteht. Er führt allerdings nur am Rande aus, welche Projekttypen auf besonders heftige Opposition stoßen und welche nicht. Es ist davon auszugehen, dass je nach Infrastrukturbereich unterschiedliche Zustimmungsraten und ein lokal unterschiedlich starkes Oppositionsverhalten zu erwarten sind. Mit Blick auf die zu untersuchenden Infrastrukturabstimmungen scheint es deshalb sinnvoll, kurz auf die spezifischen Vor- und Nachteile der lokalen Anwohnerschaft bei den drei im Zentrum stehenden Sachbereichen einzugehen.

Schweizer Bahnbauprojekte ${ }^{9}$ wie die Bahn 2000 und die Neue Eisenbahnalpentransversale (NEAT), die hauptsächlich für den öffentlichen Fern- und Güterverkehr erstellt werden, haben ihre lokalen Vorteile nicht in erster Linie in einer besseren Erschließung der Standortregion, da sie hauptsächlich Tunnelbauten und internationale IC-Strecken umfassen und damit vor allem die Durchfahrt durch die Projektregion erleichtern. Die Vorteile für die Standorte liegen vielmehr in der erhöhten Wirtschaftstätigkeit während der Bauzeit durch Aufträge von Baufirmen und lokalen Zulieferern sowie durch die Unterbringung und Ausgaben der Belegschaft der Baufirmen ${ }^{10}$. Der kurzfristigen Schaffung von Arbeitsplätzen in der Region stehen die langfristige Lärmbelastung und die Verbauung der Landschaft gegenüber.

Beim Bau von Autobahnen profitieren die Anwohner von einer erhöhten Mobilität, einer besseren Erschließung für das lokale Gewerbe und verknüpfen damit gerade in Randgebieten die Erwartung nach einer nachhaltigen Verbesserung der lokalen Wirtschafts- und Leistungskraft (Rieder 1996). Gleichzeitig kann gemäß Rieder (1996) der Autobahnbau aber durchaus auch gegenteilige Effekte ausüben. So zeigt sich, dass in einzelnen Fällen vor der Eröffnung eines neuen Autobahnabschnittes in der Schweiz die Sorge vor einem Abfluss der Kauf- und Arbeitskraft in die umliegenden städtischen Zentren groß war (Schweizerische Bundeskanzlei 1990). Aus einem ökologischen Blickwinkel erhoffen sich die Befürworter eine Entlastung der Durchfahrtsdörfer durch die Verlagerung des Verkehrs auf die Autobahn. Gegner wiederum argumentieren, dass das Verkehrsaufkommen und die Umweltbelastung in den anliegenden Kommunen dadurch noch vergrößert werden.

Atomkraftwerke zeichnen sich schließlich dadurch aus, dass sie gerade für die Standortregionen große effektive und potentielle Auswirkungen haben.

9 Die Besonderheiten von Stuttgart 21 wurden bereits in der Einleitung behandelt.

10 Strauf (2004) berechnet in ihrer Studie über den Einfluss des NEAT-Teilabschnitts in Sedrun auf die Region der Oberen Surselva einen zusätzlichen regionalen Gesamtumsatz von rund 122 Mio. CHF seit 1996. 
So schaffen AKWs durch den Bau, Betrieb und Unterhalt eine beträchtliche Zahl von Arbeitsplätzen für die Standortgemeinden ${ }^{11}$. Zudem leisten die Kraftwerke in der Regel Ausgleichsbeiträge an die Gemeinden ${ }^{12}$, bezahlen Steuern, gewährleisten eine lückenlose Energieversorgung und verbilligen den Strom für die Standortregion ${ }^{13}$ (Atomschutzverband 2009). Insgesamt führen Kernkraftwerke zu einer beträchtlichen wirtschaftlichen Wertschöpfung $^{14}$. Im Gegenzug müssen die umliegenden Gemeinden von AKWs mit erhöhten Gesundheitsrisiken und im Fall eines Reaktorunglücks mit noch deutlich größeren Folgen rechnen. So zeigt eine Studie für Deutschland, dass für Kleinkinder bis fünf Jahre die Wahrscheinlichkeit einer Krebserkrankung im Umkreis von fünf Kilometern eines AKW signifikant am höchsten ist und mit zunehmender Distanz die Häufigkeit von an Krebs erkrankenden Kleinkindern abnimmt (Kaatsch 2006). In einer Metaanalyse auf der Basis von 17 internationalen Studien kommen Baker/Hoel (2007) zudem zum Schluss, dass im näheren Umkreis von AKWs nicht nur bei Kindern, sondern auch bei Erwachsenen signifikant höhere Leukämie- und Sterblichkeitsraten festgestellt wurden. Aufgrund der stark erhöhten örtlichen Verstrahlungsrisiken bestehen in der Schweiz drei Gefahrenzonen: Zone 1 bezeichnet den Umkreis von fünf Kilometern um ein Atomkraftwerk, Zone 2 einen Perimeter von etwa 25 Kilometern, und Zone 3 steht für die übrige Schweiz. In den ersten beiden Zonen ist die Gefahr, bei einem Unfall verstrahlt zu werden, um ein Vielfaches höher als in der dritten Zone.

Insgesamt macht die kurze Beschreibung der Kosten-NutzenCharakteristika der verschiedenen Infrastrukturtypen deutlich, dass die lokalen Vor- und Nachteile ganz unterschiedlich ausfallen. Entsprechend wird in Anlehnung an Dear (1992) postuliert, dass die Ablehnung und Mobilisierung der Stimmbürger in den Standortregionen bei den drei ausgewählten Infrastrukturtypen (Straße, Bahn, AKW) verschieden sind, weshalb sie im Folgenden einzeln analysiert und danach miteinander verglichen werden. ${ }^{15}$

11 Das AKW Leibstadt beschäftigt als größtes Schweizer Kernkraftwerk rund 500 Angestellte. Die Kernkraftwerke Beznau I und II stellen sieben bis acht Prozent aller Vollzeitarbeitsplätze in den sieben umliegenden Standortgemeinden (Ecoplan 2010).

12 So zahlt z.B. das AKW Gösgen jährlich insgesamt 1,5 Mio CHF an die Standortgemeinde Däniken.

13 Das AKW Leibstadt stellt der Standortgemeinde jährlich 3,6 Mio. Kilowatt Strom rund ein Drittel des Jahresverbrauchs - unentgeltlich zur Verfügung.

14 Die ökonomische Wertschöpfung der AKWs Beznau I und II wird auf knapp 300 Mio CHF geschätzt (Ecoplan 2010).

15 Als letztes Merkmal nennt Dear (1992, S. 295) die spezifischen Eigenschaften der Infrastrukturprojekte und führt dabei verschiedene Faktoren auf wie die Größe und 
H6: Je nach Infrastrukturbereich fällt die Zustimmung der direktbetroffenen Stimmbürgerschaft unterschiedlich aus.

\section{Kontrollhypothesen}

Gemäß dem soziologischen Ansatz wird der Stimmentscheid primär mit gesellschaftlichen und kulturellen Schichtungsstrukturen erklärt (Nef 1980; Joye 1987; Voll 1991). Zwei zentrale soziokulturelle Merkmale einer Gesellschaft stellen die Sprache und die Konfession dar, die im Folgenden als Kontrollvariablen verwendet werden. Aufgrund der unterschiedlichen Einstellungen der französisch- und deutschsprachigen Schweiz zu Umweltschutzanliegen kann deshalb auch die Richtung des Stimmverhaltens vermutet werden (Hermann u. Leuthold 2002). So stellen Linder (2008, S. 144) und Vatter u.a. (2000) bei Schienen- und Straßenverkehrsvorlagen eindeutige Zustimmungsunterschiede zwischen den Sprachregionen fest. Dabei sprechen sich die Romands deutlich stärker für Straßenbauprojekte zur Förderung des Individualverkehrs aus, während die Deutschschweiz eher Projekte zum Ausbau des öffentlichen Bahnverkehrs befürwortet. Ebenfalls beobachtet Linder (2008, S. 158) einen energiepolitischen Sprachgraben in der Schweiz ${ }^{16}$.

H7: Stimmbürger französischsprachiger Kommunen stimmen stärker für Autobahnund AKW-Projekte und stärker gegen Eisenbahnprojekte als die Stimmbürger der Deutschschweiz.

H8: Stimmbürger aus katholischen Gemeinden stimmen bei Infrastrukturvorlagen anders als Stimmbürger aus protestantischen Gemeinden.

In Bezug auf die soziostrukturellen Merkmale ist im Weiteren die Unterscheidung zwischen Zentrums- und Peripherieregionen bedeutsam. So stellt Steffen (2002, S. 12) ein unterschiedliches Abstimmungsverhalten in Bezug auf Fragen der Mobilität zwischen Stimmbürgern aus Zentrums- bzw. Ag-

Anzahl der Anlagen, ihre Sichtbarkeit und ihren Ruf. Zwar können wir diese Eigenschaften aufgrund der fehlenden Varianz zwischen den einzelnen Bauprojekten innerhalb eines Sachbereichs nicht berücksichtigen. Um jedoch den Einfluss durch projektspezifische Faktoren abzuschätzen, werden die einzelnen Abstimmungsvorlagen zusätzlich als Dummy-Variablen in die Analyse aufgenommen.

16 Zur S21-Abstimmung lässt sich ebenfalls eine politisch-kulturelle These formulieren. Der Zusammenschluss Badens mit Württemberg-Baden und WürttembergHohenzollern 1952 zum Bundesland Baden-Württemberg führte vor allem auf badischer Seite zu Widerstand. Es kann daher vermutet werden, dass die Badener einer finanziellen Beteiligung über Landessteuern an einem rein württembergischen Bahnprojekt besonders ablehnend gegenüberstehen. 
glomerationsgemeinden und Stimmbürgern aus peripheren Landgemeinden fest. Linder (2008, S. 154) kommt zudem zum Schluss, dass sich insbesondere Stadt und Land bei energiepolitischen Abstimmungen gegenseitig blockieren. Während sich städtische Gebiete für eine Einschränkung der Atomenergie und von AKWs aussprechen, unterstützt die ländliche Gesellschaft mehrheitlich den Ausbau der Atomenergie. Es wird daher ein deutlicher Effekt des Urbanisierungsgrades angenommen.

H9: Je ausgeprägter die Urbanisierung, desto stärker stimmen die Stimmbürger gegen AKWs und Autobahn- und für Eisenbahnprojekte.

In verschiedenen Abstimmungsanalysen wird auch auf die Bedeutung der wirtschaftlichen Lage hingewiesen (Blöchliger/Spillmann 1992; Bühlmann u.a. 2006; Freitag u.a. 2003). Es ist zu vermuten, dass eine schlechtere wirtschaftliche Situation die Stimmbürger eher vorsichtig stimmen lässt und sie daher eher den status quo bevorzugen.

H10: Je geringer die wirtschaftliche Leistungskraft ist, desto stärker stimmen die Stimmbürger gegen neue Infrastrukturprojekte.

Auch neue gesellschaftliche Spannungslinien spielen eine relevante Rolle für die Erklärung des Stimmverhaltens bei Umweltschutz-, Verkehrs- und Energievorlagen (Linder u.a. 2008; Stadelmann-Steffen 2011). So ist bei diesen Vorlagen insbesondere ein Einfluss des Materialismus-PostmaterialismusKonflikts im Sinne der Wertewandelthese von Inglehart (1977) zu erwarten, weil Bürgerproteste gegen Infrastruktur- und Großtechnologieprojekte den Anfang der Politisierung des Umweltproblems - und damit mittelfristig die Entstehung der Grünen Parteien in Westeuropa - begründet haben, weshalb die Stärke der Grünen Partei ein valider Indikator für die postmaterialistischen Werteeinstellungen in einer Gemeinde darstellt. Umgekehrt steht in der Eidgenossenschaft die nationalkonservative Schweizerische Volkspartei (SVP) für einen Ausbau des privaten Straßenverkehrs zur Stärkung der Wirtschaft und Mobilität (SVP 2011a). Im Weiteren ist die SVP heute die einzige größere Partei in der Schweiz, die sich langfristig für die weitere Nutzung der Atomenergie ausspricht (SVP 2011b) und damit die Gegenposition zur Grünen Partei einnimmt. Für „Stuttgart 21" stellt sich die Situation anders dar, denn obwohl es sich um ein ÖV-Projekt handelt, wehren sich hauptsächlich die Grünen dagegen, weil sie befürchten, dass dadurch Steuermittel für andere Bahnprojekte fehlen, nicht abschätzbare Kosten- und Baurisiken bestehen, Mineralquellen verschmutzt werden und der denkmalgeschützte Stuttgarter Bahnhof zerstört wird (Die Grünen 2011).

H11a: Je höher der Wähleranteil der Grünen in einer Gemeinde ist, desto stärker werden das S21-Projekt sowie Autobahn- und AKW-Projekte abgelehnt, Schweizer Eisenbahnprojekte hingegen befürwortet. 
H11b: Je höher der Wähleranteil der SVP (respektive der CDU) in einer Gemeinde ist, desto stärker werden Autobahn- und AKW-Projekte (respektive S21) angenommen und Schweizer Eisenbahnprojekte abgelehnt.

Eine wichtige Rolle für den Stimmentscheid spielt schließlich der politische Prozess, der vorgängig zur Abstimmung stattgefunden hat. Eine zentrale Bedeutung kommt in der Schweiz dabei den politischen Parteien zu, die im Parlament die Abstimmungsvorlage beraten, danach ihre Parolen fassen, Koalitionen bilden und Kampagnen führen. Auch wenn der Einfluss der Parteiparolen auf die Stimmbürgerschaft umstritten ist (Kriesi 2005; Milic 2008), gelten sie als recht zuverlässiger Indikator für den Ausgang von Volksabstimmungen (Bühlmann u.a. 2006). Es ist deshalb davon auszugehen, dass einem Infrastrukturprojekt umso eher zugestimmt wird, je mehr Parteien sich zugunsten der Sachvorlage aussprechen.

H12: Je breiter abgestützt die parteipolitischen Zustimmungskoalitionen sind, desto höher ist der Ja-Anteil der Stimmbürgerschaft zu den Infrastrukturprojekten.

\section{Forschungsdesign und Operationalisierung}

\section{Die Untersuchungsfälle}

Die empirische Überprüfung der Hypothesen erfolgt anhand der S21Volksabstimmung sowie weiterer Infrastrukturabstimmungen aus der Schweiz mithilfe von Aggregatdaten ${ }^{17}$. Insgesamt existieren in der Schweiz drei Bereiche von materiellen Infrastrukturvorhaben, die einen überregionalen (nationalen) Nutzen bei gleichzeitig direkten (negativen und positiven) Folgen für die Standortregion aufweisen und zu denen sich die Bürgerschaft auch in Volksabstimmungen äussern kann. Es handelt sich um Autobahn-,

17 Ein alternatives Untersuchungsdesign wäre die Auswertung von Umfragedaten. Dagegen spricht allerdings, dass die vorliegenden Nachbefragungsdaten zu eidgenössischen Volksabstimmungen (VOX-Daten) in der Regel nur 500-700 effektiv Teilnehmende für die gesamte Schweiz umfassen und aufgrund der erhobenen Daten eine eindeutige Zuordnung der Befragten zu ihrer Wohngemeinde in den meisten Fällen nicht möglich ist. Zudem weisen Studien nach, dass die befragten Einstellungen und das effektive politische Verhalten der Bürger bei NIMBY-Situationen stark auseinanderfallen (Lober 1995). Schließlich stellt sich bei Umfragen zusätzlich das „overreporting“"-Problem in Bezug auf die Stimmbeteiligung (im langjährigen Durchschnitt sagen $60 \%$ der Befragten, dass sie an der Abstimmung teilgenommen haben, während die effektive Partizipationsrate bei Volksabstimmungen in der Schweiz aber nur $45 \%$ beträgt). 
Eisenbahnbauprojekte und um Atomkraftwerke. Für die Analyse wurden alle dreizehn eidgenössischen Abstimmungen zu diesen drei Themenbereichen zwischen 1980 und 2010 berücksichtigt. Die Liste der verwendeten Vorlagen findet sich im Anhang (Tabelle 5). Aus arbeitsökonomischen Gründen wurde jeweils eine Zufallsstichprobe von 750 Gemeinden aus der Grundgesamtheit von 2.905 Gemeinden gezogen. Diese 750 Gemeinden wurden für alle Vorlagen verwendet, was zu 3.745 Untersuchungseinheiten bei den Autobahn-, zu 2.247 Fällen bei den Eisenbahn- und zu 3.440 Fällen bei den AKWAbstimmungen führt. Für „Stuttgart 21“ wurden per Zufallsauswahl 500 von 1.101 Gemeinden ausgewählt.

\section{Das methodische Vorgehen}

Die Datenauswertung wird aufgrund der Datenstruktur mittels einer Mehrebenenanalyse durchgeführt, wobei die Themenbereiche einzeln untersucht werden. Je nach Datenstruktur werden dabei Zwei- respektive DreiEbenen-Modelle berechnet ${ }^{18}$. Für die Schweizer Fälle bilden die Abstimmungen in den Gemeinden die erste Ebene, die zweite Ebene die Gemeinden selbst und die dritte Ebene die Kantone; bei S21 fällt die erste Ebene weg ${ }^{19}$. Es werden für alle drei Themengebiete mehrere Modelle berechnet, um den Einfluss der Distanzvariablen von Modell zu Modell zu konkretisieren ${ }^{20}$. Wir beginnen mit der räumlichen Distanz zum Abstimmungsobjekt als unveränderter, kontinuierlicher Variable, fügen sie in einem zweiten Schritt als kategorielle Variable ein, um zu überprüfen, ob ein nichtlinearer Effekt vorliegt und welche Gemeinden als „Direktbetroffene“ bezeichnet werden können. In einem dritten Schritt werden die kantonale Durchschnittsentfernung (bzw. Kreisentfernung) zum Abstimmungsobjekt in die Analyse einbezogen und die Gemeindedistanzen in Bezug zu diesen Durchschnittswerten gesetzt. Dies soll zeigen, ob die im Modell 1 oder 2 erhaltenen Resultate auf einen Einfluss der Distanz der Kommunen innerhalb eines Kantons (respektive Kreises) oder auf die Durchschnittsdistanz eines Kantons (respektive Kreises) zurückzuführen sind. Zudem werden Interaktionsterme zwischen der kommunalen oder kantonalen (bzw. Kreis-)Stimmbeteiligung und der Dis$\operatorname{tanz}$ berechnet. Bis auf die kontinuierliche Distanzvariable wurden alle kon-

18 Für den Themenbereich „Autobahnbau“ ergibt sich im Nullmodell keine Varianz auf Abstimmungsebene, weshalb Zwei-Ebenen-Modelle geschätzt werden.

19 Eine Darstellung aller verwendeten Variablen findet sich im Anhang (Tabelle 6).

20 Ab Modell 2 werden nur noch die in Modell 1 signifikanten Variablen verwendet. 
tinuierlichen Variablen zentriert, so dass jeweils die Durchschnittswerte als Referenzfälle angesehen werden können.

\section{Die abhängige Variable}

Das Abstimmungsresultat in den Gemeinden (Ja-Stimmenanteil in \%) zu den 13 untersuchten Schweizer Abstimmungen und zu S21 bildet die abhängige Variable $^{21} . \mathrm{Zu}$ finden sind diese auf der entsprechenden Websites des Bundesamtes für Statistik (2011) und des Statistischen Landesamtes BadenWürttembergs (2011).

\section{Die zentrale erklärende Variable}

Die zentrale erklärende Variable, die räumliche Distanz, musste je nach Themenbereich unterschiedlich operationalisiert werden. Bei den Straßenbau- und Eisenbahnabstimmungen wurden jeweils detailliert die Abstimmungsvorlagen anhand der offiziellen Abstimmungsunterlagen des Bundes (Schweizerische Bundeskanzlei 2011) erfasst und daraus die Hauptprojektorte entnommen. Danach wurden mit Hilfe der Internetseite „www.luftlinie.org“ die Distanzen dieser Projektorte zu jeder einzelnen Gemeinden ermittelt. Bei den AKW-Abstimmungen wird die Distanz zwischen den Gemeinden und dem nächstgelegenen Schweizer Atomkraftwerk verwendet und zusätzlich eine Variable zur Entfernung zum nächstgelegenen projektierten, jedoch nie realisierten Atomkraftwerk gebildet. Für Stuttgart 21 schließlich wurden die Distanzen zu Stuttgart (oder Ulm) berechnet und jeweils die geringere davon verwendet.

\section{Die weiteren erklärenden Variablen}

Die Höhe der Stimmbeteiligung in Prozent stützt sich ebenfalls auf die Internetseite der Schweizerischen Bundeskanzlei (2011) und des Statistischen

21 Da der Wertebereich dieser Variable auf Werte zwischen 0 und 100 Prozent beschränkt ist, besteht bei Mehrebenenanalysen die Gefahr von Verzerrungen. Die Überprüfung mit Hilfe einer transformierten, logarithmierten Variable hat jedoch gezeigt, dass dies keine bedeutenden Auswirkungen hat. Es wird daher zugunsten einer besseren Interpretierbarkeit der Resultate auf diese Transformation verzichtet. 
Landesamtes Baden-Württemberg (2011). Sie wird in eine durchschnittliche kantonale Stimmbeteiligung und eine Gemeindestimmbeteiligung zentriert an diesem kantonalen Durchschnitt unterteilt. Die Sprachregion, der Katholikenanteil, die kantonale Bevölkerungsgröße sowie der Stimmenanteil der Grünen und der SVP bei den letzten Nationalratswahlen konnten der Datenbank des Bundesamtes für Statistik (2011) entnommen werden. Letztere wurden ebenfalls aufgeteilt in eine kantonale Durchschnittsstärke der Parteien und die Abweichung der Gemeinden von diesem Durchschnitt. Die Urbanisierung wird mittels Faktorenanalyse aus den Indikatoren Ausländeranteil, Bevölkerungsdichte, Anteil der Siedlungsfläche an der gesamten Kantonsfläche, Anteil Konfessionslose und Anteil des ersten und dritten Wirtschaftssektors gebildet. Auch zur Konstruktion der wirtschaftlichen Leistungskraft wurde eine Faktorenanalyse mit Daten derselben Datenbank durchgeführt. Der Faktor wird gebildet aus Indikatoren zur Steuerbelastung in einem Kanton, zur Erwerbsquote in einem Kanton und zum Pro-Kopf-Einkommen in einem Kanton. Bei beiden Faktorenanalysen laden alle Variablen auf je einen Faktor, was ihre Verwendung als Variable sinnvoll erscheinen lässt. Die Koalitionen schliesslich beruhen auf den Abstimmungsparolen der kantonalen Parteisektionen auf der Basis der Onlinedatenbank Swissvotes (Bolliger u.a. 2011). Die Daten zu den Kontrollvariablen zu Stuttgart 21 wurden allesamt von der Internetseite des Statistischen Landesamtes Baden-Württemberg entnommen. Auch hier wurden der Urbanisierungsgrad und die wirtschaftliche Leistungskraft mithilfe von Faktorenanalysen gebildet.

\section{Empirische Befunde}

1. Die Volksabstimmung zum Bahnprojekt Stuttgart 21 in BadenWürttemberg

Ein erster Blick auf die Volksabstimmung zum S21-Kündigungsgesetz vom 27. November 2011 macht deutlich, dass das Stimmverhalten in den 44 Stimmkreisen (Stadt- und Landkreise) und den 1.101 Gemeinden sehr stark schwankte. Während das Gesetz zur Aufkündigung der Finanzierung von insgesamt 58,9\% der Stimmenden des Landes Baden-Württemberg abgelehnt wurde, sprachen sich die sieben Kreise im weiter entfernten badischen Landesteil für das Kündigungsgesetz aus, darunter mit deutlichen Mehrheiten die badischen Universitätsstädte Freiburg, Heidelberg und Mannheim. Gleichzeitig befürworteten durch S21 begünstigte Städte wie Ulm oder Kommunen entlang der Achse Stuttgart - Singen besonders deutlich die weitere Finan- 
zierung des Projekts S21. Die am stärksten betroffene Stadt Stuttgart stimmte schließlich mit 52,9\% Nein einer Weiterverfolgung des Projekts nur knapp $\mathrm{zu}$.

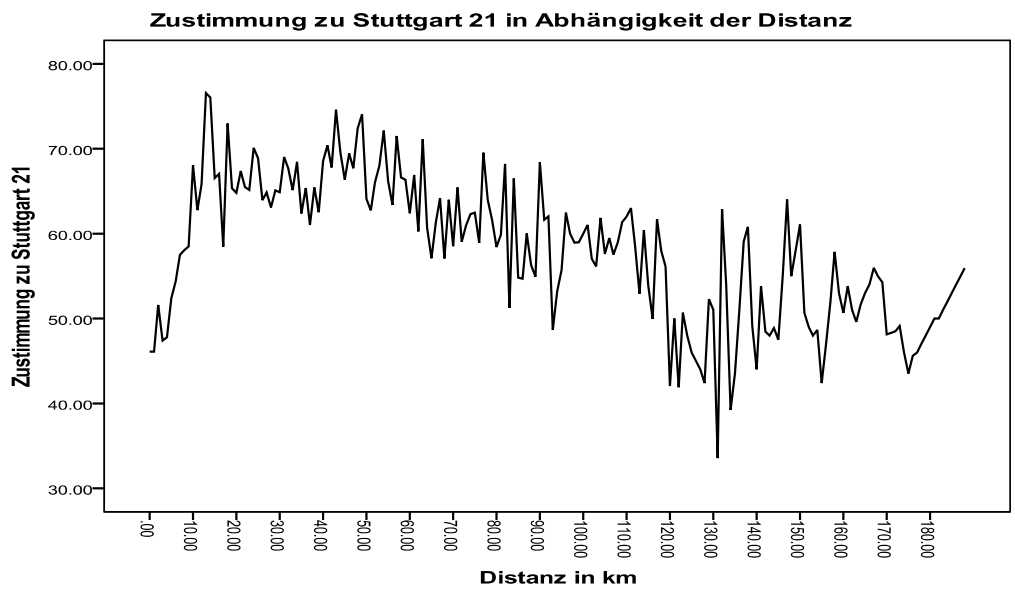

Abbildung 1 gibt die Zustimmungswerte zu Stuttgart 21 in Abhängigkeit der örtlichen Distanz wieder, wobei neben den Gemeinden Baden-Württembergs zusätzlich auch noch alle 18 Stadtbezirke von Stuttgart berücksichtigt wurden, die bis zu elf Kilometer vom Hauptbahnhof entfernt liegen. Die Zustimmungsanteile folgen dabei dem erwarteten NIMBY-Verlaufsmuster: Während sich die durch den Baulärm, Tunnelbau und Trassenverlauf lokal negativ betroffenen Bezirken des inneren Stadtgebiets in unmittelbarer Nähe des Bahnhofs teilweise deutlich für eine Kündigung aussprachen, stimmten alle Stadtbezirke des äußeren (und damit durch den Baulärm nicht betroffenen) Stadtgebiets für das Projekt S21 (Schwarz 2011). Die höchste Zustimmung findet sich in 13 Kilometern Entfernung vom Hauptbahnhof, also ganz knapp außerhalb des Stadtgebiets. Danach nimmt die Ablehnung des Infrastrukturprojekts mit zunehmender Distanz kontinuierlich zu und erreicht ihre Spitze mit 66,5\% Stimmenanteil bei der rund 130 Kilometer entfernten Stadt Freiburg. Die durchgeführten multivariaten Mehrebenenanalysen auf der Basis der kommunalen Stimmenanteile bestätigen das vorgefundene Bild: Modell 1.1 mit der örtlichen Distanz als unveränderte Variable zeigt deutlich, dass die Zustimmung zu S21 mit zunehmender Distanz abnimmt ${ }^{22}$. Dasselbe

22 Das S21-Kündigungsgesetz sah die Kündigung der Finanzierung des Projekts durch das Land Baden-Württemberg vor, war also kontraintuitiv formuliert. Im Sinne eines 
Muster ergeben die kategoriellen Distanzdummies in Modell 1.2. Sie zeigen einen deutlichen Einfluss der Distanz außerhalb des Standorts, jedoch erst ab einer Entfernung von 60 Kilometern. Es spielte also eine entscheidende Rolle, wie nahe sich eine Kommune beim Standort befand. Modell 1.3 unterscheidet zusätzlich zwischen der durchschnittlichen Distanz eines Stimmkreises und der Distanz einer Gemeinde innerhalb dieses Kreises, wobei nur letztere einen signifikanten Einfluss aufweist. Nicht überaschenderweise ist die Stuttgarter Beteiligungsquote mit 67,8\% mit Abstand die höchste aller Stimmkreise, gefolgt von den umliegenden Landkreisen, während der Stadtkreis Mannheim mit 33,3\% das Schlusslicht bildet. Die Beteiligung wirkt sich dabei stark auf den Stimmentscheid aus: Je höher die Stimmbeteiligung ist, desto höher ist die Zustimmung zu Stuttgart 21.

Tabelle 1: Resultate der Mehrebenen-Analyse der Abstimmung zu Stuttgart $21^{1}$

\begin{tabular}{|c|c|c|c|c|c|}
\hline Variable & Modell 1.1 & Modell 1.2 & Modell 1.3 & Modell 1.4 & Modell 1.5 \\
\hline Distanz (allg.) & $-0.037 * * *$ & & & & \\
\hline Distanz: $21-40 \mathrm{~km}^{2}$ & & -0.631 & & & \\
\hline Distanz: 41-60 km & & -0.858 & & & \\
\hline Distanz: $61-80 \mathrm{~km}$ & & $-2.313 * *$ & & & \\
\hline Distanz: 81-110 km & & $-4.164 * * *$ & & & \\
\hline Distanz: $111-140 \mathrm{~km}$ & & $-3.754 * *$ & & & \\
\hline Distanz: ab $141 \mathrm{~km}$ & & $-3.212 *$ & & & \\
\hline $\operatorname{Distan}^{\mathrm{G}}$ & & & $-0.035 * *$ & $-0.037 * *$ & $-0.035 * *$ \\
\hline Distanz $^{\mathrm{K}}$ & & & -0.029 & -0.029 & -0.128 \\
\hline Beteiligung $^{\mathrm{G}}$ & $0.112 * * *$ & $0.114 * * *$ & $0.112 * * *$ & $0.107 * * *$ & $0.112 * * *$ \\
\hline Beteiligung $^{\mathrm{K}}$ & $0.389 * * *$ & $0.347 * * *$ & $0.375 * * *$ & $0.376 * * *$ & $0.293 * *$ \\
\hline Beteiligung $^{\mathrm{G} *}$ Distanz ${ }^{\mathrm{G}}$ & & & & 0.003 & \\
\hline Beteiligung $^{\mathrm{K}} *$ Distanz $^{\mathrm{K}}$ & & & & & 0.002 \\
\hline Stärke Grüne ${ }^{\mathrm{G}}$ & $-0.707 * * *$ & $-0.683 * * *$ & $-0.708 * * *$ & $-0.704 * * *$ & $-0.708 * * *$ \\
\hline Stärke Grüne ${ }^{\mathrm{K}}$ & $-0.591 * * *$ & $-0.652 * * *$ & $-0.659 * * *$ & $-0.666 * * *$ & $-0.680 * * *$ \\
\hline Stärke CDU & $0.322 * * *$ & $0.332 * * *$ & $0.321 * * *$ & $0.320 * * *$ & $0.321 * * *$ \\
\hline Stärke $\mathrm{CDU}^{\mathrm{K}}$ & $0.637 * * *$ & $0.694 * * *$ & $0.660 * * *$ & $0.654 * * *$ & $0.641 * * *$ \\
\hline
\end{tabular}

einfacheren Verständnisses und in Übereinstimmung mit den weiteren Projekten wird die Variable ,gedreht“. Somit entspricht eine höhere Prozentzahl einer größeren Zustimmung zum Projekt Stuttgart 21. 


\begin{tabular}{|c|c|c|c|c|c|}
\hline $\begin{array}{l}\text { Veränderung } \\
\text { Parteienstärke }\end{array}$ & $-0.086 * *$ & $-0.097 * *$ & $-0.086 * *$ & $-0.086 * *$ & $-0.086 * *$ \\
\hline $\begin{array}{l}\text { Veränderung } \\
\text { Parteienstärke }\end{array}$ & 0.055 & 0.056 & 0.041 & 0.039 & 0.071 \\
\hline Bevölkerungsgröß $\mathrm{e}^{3}$ & -4.736 & & & & \\
\hline $\begin{array}{l}\text { Wirtschaftliche } \\
\text { Leistungskraft }\end{array}$ & -0.059 & & & & \\
\hline Urbanisierungsgrad & -0.348 & & & & \\
\hline Intercept & $66.859 * * *$ & $65.180 * * *$ & $63.200 * * *$ & $63.217 * * *$ & $56.461 * * *$ \\
\hline Residuen & $10.261 * * *$ & $10.090 * * *$ & $10.260 * * *$ & $10.222 * * *$ & $10.253 * * *$ \\
\hline Kreis-Varianz & $1.871 * *$ & $1.969 * *$ & $2.231 * *$ & $2.218 * *$ & $2.141 * *$ \\
\hline $\mathrm{AIC}$ & 2656 & 2653 & 2657 & 2657 & 2657 \\
\hline $\mathrm{N}$ & 500 & 500 & 500 & 500 & 500 \\
\hline
\end{tabular}

$*<0.05, * *<0.01, * * *<0.001$

${ }^{1}$ Die Resultate wurden ,gedreht“, die Resultate bezeichnen die Zustimmung zu Stuttgart 21, nicht zum S21-Kündigungsgesetz, ${ }^{2}$ RK: Distanz: 0-20 km, ${ }^{3}$ in $100 ` 000,{ }^{4}$ bedeutet: Grünen-Gewinne X CDU-Verluste

${ }^{\mathrm{G}}$ Gemeindewerte, zentriert am Kreisdurchschnitt, ${ }^{\mathrm{K}}$ Durchschnitt des Kreises.

Da die Distanz und die Stimmbeteiligung zudem stark korrelieren ${ }^{23}$, wurde in Modell 1.4 ein Interaktionsterm dieser beiden Variablen hinzugefügt. Dadurch erhält man für die Distanz der Kommunen bezüglich der durchschnittlichen Kreisdistanz einen negativen, sich mit zunehmender Stimmbeteiligung abschwächenden Effekt. Näher gelegene Kommunen stimmten also stärker für S21 als weiter entfernte, wobei sich die Distanz nur bei einer mittleren Beteiligung signifikant auswirkt (vgl. Abb. 1.1 bis 1.4 im Anhang). Bemerkenswerterweise wirkte sich die Kreis-Beteiligung ab einer gewissen Entfernung von Stuttgart oder Ulm signifikant positiv auf die Zustimmung zu S21 aus, während sie ansonsten keinen signifikanten Einfluss ausübte. Somit wurden ab einer gewissen Distanz vor allem Projektbefürworter mobilisiert. Mit Blick auf die zahlreichen Kontrollvariablen zeigt sich wie erwartet ein besonders deutlich negativer (respektive positiver) Einfluss der Stärke der Grünen (respektive der CDU) auf die Höhe der Zustimmung zu S21 in einer Kommune, was die starke parteipolitische Überlagerung der Stuttgart 21-Positionen bei den Stimmberechtigten zum Ausdruck bringt ${ }^{24}$. Interessan-

23 Der Korrelationskoeffizient beträgt -0.61 und ist auf dem 1\%-Niveau signifikant.

24 Die beiden stärksten Variablen geographische Distanz und Stärke der Grünen erklären allein 69\% der Varianz der kommunaler Stimmresultate in Baden-Württemberg. 
terweise üben zusätzlich auch die Wählergewinne und -verluste zwischen diesen beiden Parteien einen bedeutsamen Effekt aus: Je stärker die Grünen in einer Kommune bei den Landtagswahlen 2011 auf Kosten der CDU zugelegt haben, desto stärker stimmte die Gemeinde gegen Stuttgart 21 und umgekehrt. Dieses Ergebnis weist auf eine gewisse Relevanz des plebiszitären S21-Charakters der letzten Landtagswahlen hin. Keinen signifikanten Einfluss haben dagegen etwa der Urbanisierungsgrad, die Bevölkerungsgröße sowie die wirtschaftliche Leistungskraft, was bisherige Befunde zu S21 bestätigen, die ebenfalls eher geringe soziostrukturelle Effekte feststellten (Schwarz 2011, Wagschal 2012). Eigene weiterführende (hier nicht abgebildete) Analysen zeigen schließlich, dass zwar die Kreise und Gemeinden des badischen Landesteils S21 signifikant stärker abgelehnt haben. Wird aber gleichzeitig für die Stärke der Grünen kontrolliert, erweist sich die „Baden“Variable als nicht mehr signifikant. Mit anderen Worten: Da die Badener signifikant stärker grün wählen als die Württemberger, ist der kulturelle Effekt in der multivariaten Analyse nicht mehr bedeutsam.

\section{Die Volksabstimmungen zu den Bahnprojekten in der Schweiz}

Bei den Abstimmungen über große Bahnprojekte in der Schweiz wirkt die kontinuierliche Distanz im Modell 2.1 signifikant positiv auf den Stimmentscheid. Die Zustimmung zu Eisenbahnprojekten ist in den direktbetroffenen Gebieten ceteris paribus rund 6,7 Prozentpunkte niedriger als in Kommunen in 100 Kilometern Entfernung und mehr. Auch die kategoriellen Distanzvariablen in Modell 2.2 zeugen von diesem Effekt: Die Stimmbürger in Gemeinden ab 40 Kilometer verfügen offensichtlich über eine signifikant andere Kosten-Nutzen-Bilanz als diejenigen in den direkt umliegenden Gemeinden, da letztere den Bahnprojekten zwischen drei und zehn Prozentpunkte signifikant schwächer zustimmen. Doch auch bei größerer Entfernung steigt die Zustimmung weiter an. Die Volksentscheide zu neuen Bahnprojekten in der Schweiz scheinen somit eine Situation darzustellen, bei der die Anwohner der Projektstandorte die neuen Bahnlinien wie die Bahn 2000 und die NEAT in erster Linie als eine öffentliche Infrastrukturleistung betrachten, deren lokale Nachteile für die Direktbetroffenen höher ausfallen als der gesamtgesellschaftliche Ertrag. Auffallend ist dabei, dass der Einfluss der Distanz nicht lokal begrenzt ist, sondern auch noch bei weiter entfernten Gemeinden auftritt. In Modell 2.3 zeigt sich, dass dieser Effekt nicht durch die Distanz einer Gemeinde zur kantonalen Durchschnittsdistanz bedingt ist, sondern durch die durchschnittliche Distanz eines Kantons. Weiter wird 
deutlich, dass eine höhere Stimmbeteiligung sowohl auf Gemeinde- als auch auf Kantonsebene zu einer stärkeren Ablehnung der Bahnprojekte führt. Bei Bahnprojekten ist die Korrelation zwischen Distanz und Stimmbeteiligung nur schwach, jedoch hoch signifikant ${ }^{25}$. Wird nun in Modell 2.4 ein Interaktionsterm für Gemeindedistanz und Gemeindebeteiligung eingefügt, verliert die Gemeindedistanz ihren signifikanten Einfluss. In Modell 2.5 ergibt sich durch den Interaktionsterm für die Kantonsdistanz ein ausschliesslich positiver Einfluss, der bis auf Kantone mit sehr niedriger Stimmbeteiligung stark signifikant ist. Die Distanz vergrössert also die Zustimmung zu den Bahnprojekten, wobei eine hohe Stimmbeteiligung diesen Effekt noch verstärkt (vgl. Abb. 2.1 bis 2.4 im Anhang).

Tabelle 2: Resultate der Mehrebenen-Analyse der Bahn-Abstimmungen

\begin{tabular}{|c|c|c|c|c|c|c|c|c|c|c|}
\hline Variable & Modell 2 & & Modell 2.2 & & Modell 2. & & Modell 2 & & Modell 2 & \\
\hline Distanz (allg.) & 0.067 & $* * *$ & & & & & & & & \\
\hline $\begin{array}{l}\text { Distanz: 6-20 } \\
\mathrm{km}^{1}\end{array}$ & & & 0.426 & & & & & & & \\
\hline $\begin{array}{l}\text { Distanz: 21-40 } \\
\text { km }\end{array}$ & & & 1.402 & & & & & & & \\
\hline $\begin{array}{l}\text { Distanz: 41-60 } \\
\text { km }\end{array}$ & & & 3.083 & $*$ & & & & & & \\
\hline $\begin{array}{l}\text { Distanz: } 61-100 \\
\mathrm{~km}\end{array}$ & & & 4.791 & $* * *$ & & & & & & \\
\hline $\begin{array}{l}\text { Distanz: 101- } \\
140 \mathrm{~km}\end{array}$ & & & 10.656 & $* * *$ & & & & & & \\
\hline $\begin{array}{l}\text { Distanz: ab } 141 \\
\text { km }\end{array}$ & & & 7.961 & $* * *$ & & & & & & \\
\hline $\operatorname{Distanz}^{\mathrm{G}}$ & & & & & 0.030 & & 0.029 & & 0.030 & \\
\hline $\operatorname{Distan}^{\mathrm{K}}$ & & & & & 0.080 & $* * *$ & 0.080 & $* * *$ & 0.086 & $* * *$ \\
\hline Beteiligung $^{\mathrm{G}}$ & -0.094 & $* * *$ & -0.087 & $* *$ & -0.103 & $* * *$ & -0.104 & $* * *$ & -0.103 & $* * *$ \\
\hline Beteiligung $^{\mathrm{K}}$ & -0.406 & $* * *$ & -0.438 & $* * *$ & -0.422 & $* * *$ & -0.423 & $* * *$ & -0.402 & $* * *$ \\
\hline $\begin{array}{l}\text { Beteiligung }^{\mathrm{G}} * \\
\text { Distanz }^{\mathrm{G}}\end{array}$ & & & & & & & -0.001 & & & \\
\hline $\begin{array}{l}\text { Beteiligung }^{\mathrm{K}} * \\
\text { Distanz }^{\mathrm{K}}\end{array}$ & & & & & & & & & 0.002 & \\
\hline
\end{tabular}

25 Der Korrelationskoeffizient beträgt -0.10 und ist auf dem 1\%-Niveau signifikant. 


\begin{tabular}{|c|c|c|c|c|c|c|c|c|c|c|}
\hline $\begin{array}{l}\text { Sprache: } \\
\text { französisch }\end{array}$ & 1.329 & & 1.234 & & 1.494 & & 1.474 & & 1.523 & \\
\hline $\begin{array}{l}\text { Sprache: } \\
\text { italienisch }\end{array}$ & 8.256 & $* *$ & 8.856 & $* *$ & 7.835 & $*$ & 7.748 & * & 7.884 & $*$ \\
\hline $\begin{array}{l}\text { Sprache: } \\
\text { rätoromanisch }\end{array}$ & -0.130 & & -0.017 & & -0.097 & & -0.279 & & -0.080 & \\
\hline Stärke GPS $^{\mathrm{G}}$ & 0.296 & $* *$ & 0.279 & $* *$ & 0.299 & $* *$ & 0.302 & $* *$ & 0.298 & $* *$ \\
\hline Stärke GPS ${ }^{\mathrm{K}}$ & -0.383 & $* * *$ & -0.286 & $* *$ & -0.302 & $*$ & -0.308 & $*$ & -0.299 & * \\
\hline Stärke SVP ${ }^{\mathrm{G}}$ & -0.237 & $* * *$ & -0.241 & $* * *$ & -0.236 & $* * *$ & -0.237 & $* * *$ & -0.236 & $* * *$ \\
\hline Stärke SVP ${ }^{\mathrm{K}}$ & 0.075 & & 0.079 & & 0.081 & & 0.082 & & 0.085 & \\
\hline $\begin{array}{l}\text { Wirtschaftliche } \\
\text { Leistungskraft }\end{array}$ & -3.991 & $* * *$ & -4.046 & $* * *$ & -4.265 & $* * *$ & -4.277 & $* * *$ & -4.255 & $* * *$ \\
\hline $\begin{array}{l}\text { Urbanisie- } \\
\text { rungsgrad }\end{array}$ & -0.647 & & & & & & & & & \\
\hline $\begin{array}{l}\text { Bevölkerungs- } \\
\text { größ } \mathrm{e}^{3}\end{array}$ & 0.839 & & & & & & & & & \\
\hline $\begin{array}{l}\text { Anteil } \\
\text { Katholiken }\end{array}$ & 0.028 & & & & & & & & & \\
\hline $\begin{array}{l}\text { Koal. Bürg. vs. } \\
\text { Linke }^{4}\end{array}$ & -13.468 & $* * *$ & -13.949 & $* * *$ & -13.404 & $* * *$ & -13.406 & $* * *$ & -12.899 & $* * *$ \\
\hline $\begin{array}{l}\text { Koal. alle gg. } \\
\text { GPS }\end{array}$ & 2.267 & & 2.039 & $* * *$ & 1.790 & $* *$ & 1.802 & $* *$ & 1.316 & \\
\hline $\begin{array}{l}\text { Koal. alle gg. } \\
\text { SVP }\end{array}$ & -4.774 & $* * *$ & -4.908 & $* * *$ & -4.574 & $* * *$ & -4.583 & $* * *$ & -4.545 & $* * *$ \\
\hline Koal. SVP/FDP & -5.280 & $* * *$ & -5.115 & $* * *$ & -4.794 & $* * *$ & -4.788 & $* * *$ & -4.883 & $* * *$ \\
\hline Koal. sonstige & -17.759 & $* * *$ & -17.637 & $* * *$ & -16.951 & $* * *$ & -16.964 & $* * *$ & -16.460 & $* * *$ \\
\hline Nr. $348^{5}$ & -0.191 & & & & & & & & & \\
\hline Nr. 382 & -0.218 & & & & & & & & & \\
\hline Intercept & 56.282 & $* * *$ & 55.458 & $* * *$ & 58.092 & $* * *$ & 58.094 & $* * *$ & 58.105 & $* * *$ \\
\hline Residuen & 71.236 & $* * *$ & 70.554 & $* * *$ & 71.173 & $* * *$ & 71.222 & $* * *$ & 71.213 & $* * *$ \\
\hline $\begin{array}{l}\text { Kantonale } \\
\text { Varianz }\end{array}$ & 43.947 & $* *$ & 42.426 & $* *$ & 44.070 & $* *$ & 44.391 & $* *$ & 43.761 & $* *$ \\
\hline $\begin{array}{l}\text { Gemeinde- } \\
\text { varianz }\end{array}$ & 35.182 & $* * *$ & 35.257 & $* * *$ & 35.063 & $* * *$ & 34.924 & $* * *$ & 35.218 & $* * *$ \\
\hline AIC & 16524 & & 16558 & & 16562 & & 16564 & & 16561 & \\
\hline $\mathrm{N}$ & 2247 & & 2247 & & 2247 & & 2247 & & 2247 & \\
\hline
\end{tabular}

$*<0.05, * *<0.01, * * *<0.001$

${ }^{\mathrm{G}}$ Gemeindewerte, zentriert an kantonalem Durchschnitt, ${ }^{\mathrm{K}}$ kantonaler Durchschnitt.

${ }^{1}$ RK: Distanz: 0-5km, ${ }^{2}$ RK: Sprache: deutsch, ${ }^{3}$ in $100.000,{ }^{4}$ RK: Koalition: große Koalition aller 5 Parteien, ${ }^{5} \mathrm{RK}: \mathrm{Nr} .445$ 
Bei den Kontrollgrößen zeigt sich, dass die Zustimmung zu den Vorlagen umso höher ist, je stärker die Grünen in der Gemeinde sind bzw. je schwächer die SVP ist. Die Bevölkerungsgröße, die Urbanisierung und der Katholikenanteil sowie die Zugehörigkeit zum französischsprachigen Landesteil weisen hingegen keinen signifikanten Einfluss auf die Zustimmungsunterschiede aus. Relevant scheinen jedoch die Koalitionen der unterstützenden Parteien zu sein, denn je nach Koalition verändert sich die Zustimmung um fast 20 Prozentpunkte. Am höchsten ist die Zustimmung in Kantonen mit einer grossen Parteienkoalition ohne die Grünen, mit am niedrigsten in den Kantonen mit einer bürgerlichen Koalition.

\section{Die Volksabstimmungen zu den Autobahnprojekten in der Schweiz}

Einen Hauptbefund der Analyse zu den Schweizer Autobahn-Abstimmungen stellt der signifikant negative Einfluss der räumlichen Distanz auf den Stimmentscheid dar. So stimmen die Bürger in der nächsten Umgebung eines Autobahnbauprojekts ceteris paribus um 1,4 Prozentpunkte diesen Vorlagen stärker zu, als wenn sie sich 100 Kilometer davon entfernt befinden. Autobahnprojekte werden offensichtlich als Infrastrukturleistungen mit lokal stärkeren Vor- als Nachteilen bewertet, was in Übereinstimmung mit dem YIMBY-Phänomen steht. Wie theoretisch erwartet, ist nur eine begrenzte Reichweite des Distanzeinflusses sichtbar: So stimmen in erster Linie die Bürger der Gemeinden bis zu 20 Kilometern Entfernung den Autobahnprojekten signifikant zwischen eins und zwei Prozentpunkte stärker zu als die übrigen Regionen. Ab einer Distanz von 20 Kilometern verändert sich die Zustimmung nicht mehr systematisch. Modell 3.3 zeigt, dass sowohl innerhalb der Kantone als auch zwischen den Kantonen ein negativer Einfluss der Distanz auf den Stimmentscheid besteht. Die Distanz scheint also insgesamt einen bedeutsamen Effekt zu haben: Stimmbürger in der Nähe beurteilen Autobahnen als vorteilhafter. Dabei wirkt sich die Höhe der Stimmbeteiligung zwischen den Kantonen signifikant negativ auf den Stimmentscheid aus, d.h. bei niedriger Stimmbeteiligung ist die Zustimmung zu Autobahnprojekten deutlich höher.

Tabelle 3: Resultate der Mehrebenen-Analyse der Autobahnbau-Abstimmungen

\begin{tabular}{llllll}
\hline Variable & Modell 3.1 & Modell 3.2 & Modell 3.3 & Modell 3.4 & Modell 3.5 \\
\hline Distanz (allg.) & $-0.014 \quad * * *$ & & & \\
Distanz: $21-40 \mathrm{~km}^{1}$ & & & $-1.403 \quad * *$ &
\end{tabular}




\begin{tabular}{|c|c|c|c|c|c|c|c|c|c|c|}
\hline Distanz: 41-60 km & & & -1.801 & $* * *$ & & & & & & \\
\hline Distanz: $61-80 \mathrm{~km}$ & & & -1.296 & $*$ & & & & & & \\
\hline Distanz: $81-110 \mathrm{~km}$ & & & -1.881 & $* *$ & & & & & & \\
\hline Distanz: $111-140 \mathrm{~km}$ & & & -1.265 & & & & & & & \\
\hline Distanz: ab $141 \mathrm{~km}$ & & & -2.671 & $* * *$ & & & & & & \\
\hline $\operatorname{Distan}^{\mathrm{G}}$ & & & & & -0.020 & $*$ & -0.018 & $*$ & -0.021 & $*$ \\
\hline Distanz $z^{\mathrm{K}}$ & & & & & -0.012 & $*$ & -0.012 & $*$ & -0.011 & $*$ \\
\hline Beteiligung $^{\mathrm{G}}$ & 0.011 & & 0.010 & & 0.013 & & 0.012 & & 0.013 & \\
\hline Beteiligung $^{\mathrm{K}}$ & -0.503 & $* * *$ & -0.5491 & $* * *$ & -0.491 & $* * *$ & -0.488 & $* * *$ & -0.506 & $* * *$ \\
\hline $\begin{array}{l}\text { Beteiligung }^{\mathrm{G}} \text { * } \\
\text { Distanz }^{\mathrm{G}}\end{array}$ & & & & & & & -0.003 & $* * *$ & & \\
\hline $\begin{array}{l}\text { Beteiligung }^{\mathrm{K}} * \\
\text { Distanz }^{\mathrm{K}}\end{array}$ & & & & & & & & & -0.001 & $*$ \\
\hline $\begin{array}{l}\text { Sprache: } \\
\text { französisch }\end{array}$ & 13.302 & $* * *$ & 13.199 & $* * *$ & 13.237 & $* * *$ & 13.369 & $* * *$ & 13.233 & $* * *$ \\
\hline Sprache: italienisch & 3.059 & & 2.620 & & 2.804 & & 2.315 & & 2.79 & \\
\hline $\begin{array}{l}\text { Sprache: } \\
\text { rätoromanisch }\end{array}$ & 0.888 & & 0.438 & & 0.616 & & 0.514 & & 0.611 & \\
\hline Stärke GPS ${ }^{\mathrm{G}}$ & -0.284 & $* * *$ & -0.295 & $* * *$ & -0.292 & $* * *$ & -0.292 & $* * *$ & -0.291 & $* * *$ \\
\hline Stärke GPS ${ }^{\mathrm{K}}$ & -0.155 & & -0.028 & & -0.036 & & -0.052 & & 0.011 & \\
\hline Stärke SVP ${ }^{\mathrm{G}}$ & 0.019 & & & & & & & & & \\
\hline Stärke SVP ${ }^{\mathrm{K}}$ & -0.214 & & & & & & & & & \\
\hline $\begin{array}{l}\text { Wirtschaftliche Leis- } \\
\text { tungskraft }\end{array}$ & 9.340 & $* * *$ & 9.570 & $* * *$ & 9.673 & $* * *$ & 9.656 & $* * *$ & 10.271 & $* * *$ \\
\hline Urbanisierungsgrad & -1.854 & & & & & & & & & \\
\hline Bevölkerungsgröße $\mathrm{e}^{3}$ & -1.302 & $*$ & -1.369 & * & -1.337 & $*$ & -1.326 & $*$ & -1.490 & $*$ \\
\hline Anteil Katholiken & -0.156 & & & & & & & & & \\
\hline Koal. Alle gg. GPS ${ }^{4}$ & 3.611 & $* * *$ & 3.839 & $* * *$ & 3.694 & $* * *$ & 3.667 & $* * *$ & 3.818 & $* * *$ \\
\hline Koal. SVP/FDP & -0.176 & & -0.376 & & -0.277 & & -0.321 & & -0.656 & \\
\hline Koal. sonstige & -17.184 & $* * *$ & -17.176 & $* * *$ & -17.185 & $* * *$ & -17.388 & $* * *$ & -17.245 & $* * *$ \\
\hline Nr. $359^{5}$ & 4.153 & $* * *$ & 4.180 & $* * *$ & 4.203 & $* * *$ & 4.222 & $* * *$ & 4.254 & $* * *$ \\
\hline Nr. 360 & 2.564 & $* * *$ & 2.586 & $* * *$ & 2.564 & $* * *$ & 2.582 & $* * *$ & 2.699 & $* * *$ \\
\hline Nr. 361 & 3.073 & $* * *$ & 3.047 & $* * *$ & 3.083 & $* * *$ & 3.107 & $* * *$ & 3.049 & $* * *$ \\
\hline Nr. 504 & -34.969 & $* * *$ & -34.895 & $* * *$ & -35.127 & $* * *$ & -34.959 & $* * *$ & -35.165 & $* * *$ \\
\hline Intercept & 67.255 & $* * *$ & 67.656 & $* * *$ & 66.297 & $* * *$ & 66.221 & $* * *$ & 65.913 & $* * *$ \\
\hline
\end{tabular}




\begin{tabular}{lllllllllll} 
Residuen & 68.137 & $* * *$ & 68.002 & $* * *$ & 68.187 & $* * *$ & 67.953 & $* * *$ & 68.000 & $* * *$ \\
Kantonale Varianz & 36.362 & $* *$ & 42.155 & $* *$ & 41.938 & $* *$ & 41.436 & $* *$ & 47.070 & $* *$ \\
AIC & 26098 & & 26097 & & 26098 & & 26088 & & 26093 & \\
$\mathrm{~N}$ & 3745 & & 3745 & & 3745 & & 3745 & & 3745 & \\
\hline
\end{tabular}

$*<0.05, * *<0.01, * * *<0.001$

${ }^{\mathrm{G}}$ Gemeindewerte, zentriert an kantonalem Durchschnitt, ${ }^{\mathrm{K}}$ kantonaler Durchschnitt

${ }^{1} \mathrm{RK}$ : Distanz: 0-20km, ${ }^{2} \mathrm{RK}$ : Sprache: deutsch, ${ }^{3}$ in 100.000, ${ }^{4} \mathrm{RK}$ : Koalition: Bürgerliche gegen Linke, ${ }^{5} \mathrm{RK}:$ Nr. 362

Ebenfalls korrelieren Distanz und Stimmbeteiligung ${ }^{26}$. Bei Einbezug des entsprechenden Interaktionseffekts wirken sich sowohl die Gemeindedistanz als auch die Kantonsdistanz nur noch bei überdurchschnittlich großer Stimmbeteiligung signifikant negativ aus. Bei stark betroffenen Gemeinden stimmen also diejenigen stärker für Autobahnprojekte, die in der Nähe wohnen (vgl. Abb. 3.1 bis $3.4 \mathrm{im}$ Anhang). Die französischsprachige Schweiz stimmt den Autobahnvorlagen mit über 13 Prozentpunkten stärker zu als die Deutschschweiz, was die Beobachtung von Hermann und Leuthold (2002, S. 9) bestätigt, wonach „Vorlagen zur Begrenzung [...] des motorisierten Strassenverkehrs [in der Romandie] keine Chance [haben]“. Ein Einfluss der Parteienstärken liegt für die Grünen auf Gemeindeebene vor: Innerhalb eines Kantons stimmen Gemeinden mit einem hohen Grünenwähleranteil signifikant stärker gegen Autobahn-Projekte als die übrigen Kommunen. Gleichzeitig sind es insbesondere die Gemeinden aus kleineren und wirtschaftlich leistungsstarken Kantonen, die die Vorlage überdurchschnittlich befürworten. Schließlich stimmen Gemeinden aus Kantonen, in denen eine große befürwortende Parteienkoalition nur den Grünen gegenübersteht, um fast 14 Prozentpunkte stärker für die Projekte als Gemeinden aus Kantonen, in denen die FDP und die SVP den anderen drei großen Parteien gegenüberstehen. Dass zudem die spezifischen Vorlagenmerkmale einen ganz entscheidenden Einfluss auf die Höhe der Zustimmung ausüben, machen die Vorlagendummies deutlich. Der Gegenvorschlag zur Avanti-Initiative wurde mit mehr als 36 Prozentpunkten stärker abgelehnt als die vier Volksinitiativen.

26 Der Korrelationskoeffizient beträgt -0.35 und ist auf dem 1\%-Niveau signifikant. 
4. Die Volksabstimmungen zu Atomkraftwerken in der Schweiz

Als Besonderheit finden sich bei Volksentscheiden zu Atomkraftwerken neben den durch bestehende AKWs direktbetroffenen Regionen auch eine weitere Gruppe potentiell betroffener Regionen, die durch die Nähe zu projektierten, bis heute aber nicht realisierten Atomkraftwerken besteht. Um diese Form der Betroffenheit einzubeziehen, wird die räumliche Nähe zu einem geplanten, aber bisher nicht gebauten AKW als Variable für die Gemeinden, die näher als fünf Kilometer von einem solchen entfernt sind, in die Modelle aufgenommen $^{27}$. Für Modell 4.1 lässt sich zunächst kein signifikanter $\mathrm{Zu}$ sammenhang zwischen Stimmentscheid und Distanz beobachten. Auf den ersten Blick scheint die Distanz zu den gebauten Atomkraftwerken keinen Einfluss auszuüben, ganz im Gegensatz zu den projektierten, aber nicht gebauten AKWs: Gemeinden im Umkreis von fünf Kilometern um solche stimmen signifikant um fast sieben Prozentpunkte weniger zu. Bemerkenswerterweise macht Modell 4.2 deutlich, dass bezüglich der ersten fünf Kilometer auch bei den bestehenden Atomkraftwerken ein sehr deutlicher Einfluss der Distanz besteht, allerdings gerade in die entgegengesetzte Richtung: Standortgemeinden, die sich im Umkreis von fünf Kilometern um ein bestehendes Atomkraftwerk befinden, stimmen signifikant um drei Prozentpunkte positiver als die Stimmbürger in den darauf folgenden 15 Kilometern und zwischen drei und sieben Prozentpunkten positiver als die übrigen Stimmenden. Damit handelt es sich bei den Direktbetroffenen von AKWAbstimmungen mit bis zu maximal fünf Kilometern Entfernung zum nächsten Atomkraftwerk um einen sehr eng begrenzten Kreis von Stimmbürgern, der zudem je nach Realisierungsgrad gerade entgegengesetzt ausfällt. Zusätzlich findet sich ein zweiter Kreis von etwas stärker Betroffenen, die die nächsten 15 Kilometer umfassen. Diese beiden Kreise von Betroffenen entsprechen genau der ersten (fünf Kilometer) und ungefähr der zweiten Gefahrenzone (25 Kilometer), die bei einem Reaktorunfall ungleich höheren Verstrahlungsrisiken ausgesetzt sind als die übrigen Regionen. Modell 4.3 kontrolliert zusätzlich für die Distanzeffekte zwischen Gemeinden und Kantonen, wobei keine der beiden Größen signifikant ist. Der Einfluss der räumlichen Entfernung scheint sich bei AKW-Abstimmungen also tatsächlich nur auf die ersten fünf Kilometer zu beschränken. Kantonsintern erhöht aber eine starke Stimmbeteiligung die Zustimmung zu Atomkraftwerken zusätzlich.

27 Fünf Kilometer stellten sich bei verschiedenen Tests als diejenige Entfernung mit dem stärksten Einfluss heraus. 
Zudem korrelieren auch hier die Distanz und die Stimmbeteiligung ${ }^{28}$. Unter Einbezug des Interaktionsterms wirkt sich die Distanz insbesondere dort stark negativ auf die Zustimmung aus, wo die Beteiligung besonders hoch ist. Dies ist auf Ebene der Kantone ebenfalls der Fall, jedoch noch viel stärker für weiter entfernte Kantone (vgl. Abb. 4.1 bis 4.4 im Anhang).

Im Weiteren übt die Stimmbeteiligung auf Gemeindeebene einen positiven Einfluss aus: Je stärker die Gemeinden partizipieren, desto stärker stimmen sie für die Atomkraft, wobei dieser Zusammenhang mit zunehmender Distanz schwächer wird. Bei den Kontrollgrößen ergeben sich nur teilweise die erwarteten Zusammenhänge. So stimmt die Romandie nicht signifikant anders als die Deutschschweiz, jedoch nimmt das Tessin eine sehr atomkritische Haltung ein. Wie postuliert, wirkt sich die Stärke der Grünen Partei in einer Gemeinde signifikant negativ auf die Zustimmung aus, ebenso ist der prognostizierte (positive) SVP-Effekt sichtbar. Deutliche Auswirkungen haben auch die vorlagenspezifischen Faktoren wie die Stärke der Parteienkoalitionen und die Vorlagendummies. So sprechen sich die Bürger bei einer breit abgestützten bürgerlichen Koalition in einem Kanton signifikant stärker für AKWs aus als bei einer Befürworterkoalition, die sich auf FDP und SVP beschränkt. Bei den einzelnen Vorlagen fällt auf, dass erstens bei den beiden Abstimmungen von 2003 deutlich atomfreundlicher gestimmt wurde als 1990 und 1984 und zweitens, dass die Moratoriumsinitiativen 1990 und 2003 deutlich stärker befürwortet wurden als die beiden Atomausstiegsinitiativen.

Tabelle 4: Resultate der Mehrebenen-Analyse der AKW-Abstimmungen

\begin{tabular}{|c|c|c|c|c|c|c|}
\hline Variable & Modell 4.1 & \multicolumn{2}{|c|}{ Modell 4.2} & Modell 4.3 & Modell 4.4 & Modell 4.5 \\
\hline Distanz (allg.) & -0.022 & & & & & \\
\hline Distanz: 6-20 km & & -3.739 & $*$ & & & \\
\hline Distanz: $21-40 \mathrm{~km}$ & & -7.094 & $* * *$ & & & \\
\hline Distanz: 41-60 km & & -6.321 & $* * *$ & & & \\
\hline $\begin{array}{l}\text { Distanz: 61-100 } \\
\mathrm{km}\end{array}$ & & -3.638 & & & & \\
\hline $\begin{array}{l}\text { Distanz: 101-140 } \\
\mathrm{km}\end{array}$ & & -7.209 & $* * *$ & & & \\
\hline $\begin{array}{l}\text { Distanz: ab } 141 \\
\mathrm{~km}\end{array}$ & & -5.663 & $*$ & & & \\
\hline $\operatorname{Distanz}^{\mathrm{G}}$ & & & & -0.018 & -0.018 & -0.017 \\
\hline
\end{tabular}

28 Der Korrelationskoeffizient beträgt -0.31 und ist auf dem 1\%-Niveau signifikant. 
Distanz $z^{K}$

Distanz Nichtgebaute

Beteiligung $^{\mathrm{G}}$

Beteiligung $^{\mathrm{K}}$

Beteiligung $^{\mathrm{G}}$ *

Distanz $^{\mathrm{G}}$

Beteiligung $^{\mathrm{K}} *$

Distanz $^{\mathrm{K}}$

\begin{tabular}{|c|c|c|c|c|c|c|c|c|c|c|}
\hline $\begin{array}{l}\text { Sprache: } \\
\text { französisch }^{2}\end{array}$ & -0.172 & & -0.050 & & -0.236 & & -0.124 & & -0.237 & \\
\hline $\begin{array}{l}\text { Sprache: } \\
\text { italienisch }\end{array}$ & -2.713 & $* * *$ & -3.162 & $* *$ & -2.695 & $* *$ & -3.017 & $* *$ & -2.668 & $* *$ \\
\hline $\begin{array}{l}\text { Sprache: } \\
\text { rätoromanisch }\end{array}$ & -0.541 & & -0.856 & & -0.520 & & -0.306 & & -0.521 & \\
\hline Stärke GPS ${ }^{\mathrm{G}}$ & -0.410 & $* * *$ & -0.413 & $* * *$ & -0.408 & $* * *$ & -0.407 & $* * *$ & -0.414 & $* * *$ \\
\hline Stärke GPS ${ }^{\mathrm{K}}$ & -0.170 & & -0.265 & & -0.363 & & -0.368 & & -0.366 & \\
\hline Stärke $\mathrm{SVP}^{\mathrm{G}}$ & 0.073 & $* * *$ & 0.074 & $* * *$ & 0.073 & $* * *$ & 0.071 & $* * *$ & 0.072 & $* * *$ \\
\hline Stärke $\mathrm{SVP}^{\mathrm{K}}$ & 0.223 & & 0.164 & & 0.138 & & 0.136 & & 0.131 & \\
\hline $\begin{array}{l}\text { Wirtschaftliche } \\
\text { Leistungskraft. }\end{array}$ & -1.687 & $* * *$ & -1.700 & $* * *$ & -1.705 & $* * *$ & -1.720 & $* * *$ & -1.680 & $* * *$ \\
\hline $\begin{array}{l}\text { Urbanisierungs- } \\
\text { grad }\end{array}$ & 0.152 & & & & & & & & & \\
\hline $\begin{array}{l}\text { Bevölkerungs- } \\
\text { größ } \mathrm{e}^{3}\end{array}$ & -4.701 & & & & & & & & & \\
\hline Anteil Katholiken & 0.025 & & & & & & & & & \\
\hline Koal. SVP/FDP & -2.121 & $* *$ & -2.00 & $* *$ & -2.068 & $* *$ & -2.080 & $* *$ & -2.186 & $* *$ \\
\hline Koal. Sonstige & 0.563 & & 0.548 & & 0.574 & & 0.575 & & 0.410 & \\
\hline Nr. $321^{5}$ & -5.422 & $* * *$ & -5.127 & $* * *$ & -5.183 & $* * *$ & -5.161 & $* * *$ & -5.212 & $* * *$ \\
\hline Nr. 365 & -8.910 & $* * *$ & -8.732 & $* * *$ & -8.804 & $* * *$ & -8.811 & $* * *$ & -8.817 & $* * *$ \\
\hline Nr. 366 & -15.381 & $* * *$ & -15.206 & $* * *$ & -15.277 & $* * *$ & -15.286 & $* * *$ & -15.273 & $* * *$ \\
\hline Nr. 501 & 6.614 & $* * *$ & 6.632 & $* * *$ & 6.627 & $* * *$ & 6.624 & $* * *$ & 6.586 & $* * *$ \\
\hline Intercept & 63.584 & $* * *$ & 68.078 & $* * *$ & 62.589 & $* * *$ & 62.543 & $* * *$ & 62.730 & $* * *$ \\
\hline Residuen & 64.359 & $* * *$ & 63.824 & $* * *$ & 64.394 & $* * *$ & 64.179 & $* * *$ & 64.322 & $* * *$ \\
\hline Kantonale Varianz & 26.478 & $* *$ & 23.624 & $* *$ & 22.858 & $* * *$ & 23.024 & $* * *$ & 23.214 & $* * *$ \\
\hline Gemeindevarianz & 20.110 & $* * *$ & 19.218 & $* * *$ & 20.205 & $* * *$ & 20.203 & $* * *$ & 20.202 & $* * *$ \\
\hline
\end{tabular}

$\begin{array}{lllllllll}-7.000 & * * * & -6.521 & * * * & -7.041 & * * * & -6.932 & * * * & -6.734 \\ 0.067 & * * * & 0.068 & * * * & 0.068 & * * * & 0.068 & * * * & 0.068 \\ -0.028 & & -0.026 & & -0.036 & & -0.032 & & -0.002\end{array}$

$-0.004 \quad * * *$

0.002 


\begin{tabular}{llllll} 
AIC & 24723 & 24679 & 24719 & 24711 & 24718 \\
$\mathrm{~N}$ & 3440 & 3440 & 3440 & 3440 & 3440 \\
\hline
\end{tabular}

$*<0.05, * *<0.01, * * *<0.001$

${ }^{\mathrm{G}} \mathrm{Gemeindewerte,} \mathrm{zentriert} \mathrm{an} \mathrm{kantonalem} \mathrm{Durchschnitt,}{ }^{\mathrm{K}}$ kantonaler Durchschnitt.

${ }^{1}$ RK: Distanz: 0-5km, ${ }^{2}$ RK: Sprache: deutsch, ${ }^{3}$ in $100 ` 000,{ }^{4}$ RK: Koalition: Koalition aller fünf Parteien, ${ }^{5}$ RK: Nr. 502

\section{Schlussfolgerungen}

Führt die unmittelbare Volksgesetzgebung dazu, dass die Stimmbürger bei der Realisierung großer Infrastrukturprojekte wie Bahnhöfe, Bahnstreckenlinien, Autobahnen und Atomkraftwerke nach dem Sankt-Florians-Prinzip entscheiden? Die sich auf empirische Fakten stützende Antwort zur Überprüfung des NIMBY-Phänomens bei Volksabstimmungen fällt einerseits eindeutig aus, verlangt aber andererseits nach Differenzierungen.

Offensichtlich ist die geographische Distanz zum jeweiligen Standort eine äußerst bedeutsame Erklärungsgröße für das Stimmverhalten bei großen Infrastrukturanlagen. In allen durchgeführten Analysen erweist sich die örtliche Nähe der Stimmbürger als zentrale Determinante, womit sich die generelle Relevanz des NIMBY-Effekts bei den untersuchten Volksentscheiden bestätigt. Gleichzeitig fallen die Distanzwirkungen je nach Infrastrukturbereich aber sehr unterschiedlich aus. Während bei Abstimmungen zu Autobahnen die regional perzipierten positiven Effekte überwiegen, unterliegen Volksentscheide zu den Schweizer Bahnprojekten einem weiträumigen NIMBYPhänomen. So weist die räumliche Distanz bei den Bahnabstimmungen einen signifikant positiven Einfluss auf den Stimmentscheid aus. Hier gehen die Stimmbürger der Standortgemeinden durch den Bau einer neuen Bahnstrecke von lokal stärkeren Nachteilen aus, während Regionen ab 40 Kilometern Entfernung den Nutzen deutlich positiver beurteilen.

Eine doppelte, gleichzeitig sehr lokale als auch weit entfernte Ablehnung zeichnet schließlich den Stimmentscheid zu Stuttgart 21 aus: So lehnen sowohl die direkt betroffenen Stadtbezirke Stuttgarts in unmittelbarer Nachbarschaft zum Hauptbahnhof als auch die am weitesten entfernten badischen Regionen die weitere Finanzierung des S21-Projekts am stärksten ab. Allerdings sind die Ursachen dafür unterschiedlich. Während die dem Trassenverlauf, Tunnelbau und Baulärm direkt ausgesetzten Bezirke im Stuttgarter Stadtzentrum dem typischen NIMBY-Muster lokal negativer Betroffenheit entsprechen, lehnten die weit entfernten Regionen des badischen Landesteils die Finanzierung von S21 ab, weil sie befürchten mussten, dass bei einer 
Annahme der Vorlage keine weiteren Landesfinanzmittel für ihre eigenen lokalen Bahnprojekte zur Verfügung stehen würden (Schwarz 2011). Gleichzeitig findet sich zwischen diesen beiden Vetogürteln ein Perimeter hoher Zustimmung im Umkreis zwischen 10 bis 70 Kilometern vom Stuttgarter Hauptbahnhof entfernt, die vor allem die äußeren Stadtgebiete und die fünf Landkreise Stuttgarts umfassen und durch ihre Standortnähe überdurchschnittlich von der neuen Bahninfrastruktur von Stuttgart profitieren.

Außerhalb der Stadt Stuttgart entspricht das landesweite Stimmverhalten zu S21 eher demjenigen zu neuen Autobahnen als zu großen Bahnprojekten in der Schweiz. Dies mag damit zusammenhängen, dass S21 und die schweizerischen Bahnprojekte wie die NEAT und Bahn 2000 ganz unterschiedliche Ziele verfolgen. Während das S21-Projekt nur die Bahnhofsinfrastruktur der Landeshauptstadt sowie den Neubau einer lokal begrenzten Bahnverbindung nach Ulm umfasst, sehen die Schweizer Bahnprojekte jeweils die regional ausgewogene Realisierung neuer Bahnlinien in den verschiedenen Landesteilen der Deutsch- und Westschweiz sowie generelle Maßnahmen zum Schutz der Umwelt wie etwa die Reduktion der Lärmbelastung auf bestehenden Linien vor, die der gesamten Schweizer Bevölkerung zugutekommen (Linder u.a. 2010). Ähnlich wie bei S21 ist hingegen die Ausgangslage bei den Abstimmungen zum Bau neuer Autobahnstrecken in der Schweiz. Hier profitieren ebenfalls nur einige wenige Regionen, während ein großer Teil der Bevölkerung vor allem indirekt über die Steuern die Kosten für den Bau und Unterhalt der Infrastruktur trägt.

Kennzeichnend für das Stimmverhalten bei Atomkraftwerkabstimmungen ist schließlich die sehr starke lokale Betroffenheit. So stimmen insbesondere die Bürger im Umkreis der ersten fünf Kilometer von AKWs signifikant anders $\mathrm{ab}$ als die Stimmenden weiter entfernter Gemeinden. Allerdings entscheiden die Bürger in der nächsten Nähe zu bereits bestehenden AKWs gerade entgegengesetzt zu denjenigen, bei denen ein AKW in Planung ist (oder war): Im Fall bereits gebauter Kernkraftwerke führen offensichtlich der wirtschaftlich konkrete Nutzen (Arbeitsplätze, niedrige Steuern, Ausgleichszahlungen) bzw. die Furcht vor den negativen Konsequenzen bei der Stilllegung des AKWs zu einem überdurchschnittlich hohen Pro-AKW-Votum der Stimmbürger in den Standortgemeinden, während im Fall der projektierten Atomkraftwerke die befürchteten Nachteile wie erhöhte Umwelt- und Gesundheitsrisiken den Stimmentscheid der potentiell Direktbetroffenen stark negativ beeinflussen. Das Verhalten bei Abstimmungen zu geplanten AKWs entspricht damit genau den beiden ersten postulierten NIMBY-Hypothesen, während bei den Standortgemeinden schon bestehender AKWs ein sog. YIMBY-Effekt auftritt. In Übereinstimmung mit der NIMBY-Theorie er- 
weist sich die Mobilisierung als abhängig von der örtlichen Distanz zum Projektstandort. So nimmt bei allen Vorlagen die Beteiligung mit zunehmender geographischer Entfernung ab. Zudem verstärkt die Höhe der Stimmbeteiligung für die Abstimmungen in der Schweiz den Einfluss der Distanz auf den Stimmentscheid, d.h. dass in direktbetroffenen Gebieten die Entfernung zum Standort die Zustimmung stärker beeinflusst als in nicht direktbetroffenen Gebieten.

Mit Bezug auf die Kontrollgrößen zeigen sich besonders starke und stabile Zusammenhänge zwischen den Parteistärken und den Stimmresultaten. Wie erwartet stimmen vor allem Gemeinden mit einem hohen Wähleranteil der Grünen deutlich für Projekte zur Förderung des öffentlichen Verkehrs und gegen Atomenergie, Straßenverkehr und gegen S2 ${ }^{29}$. Allein mit dem Wähleranteil der Grünen und der örtlichen Nähe zum Standort lassen sich bei einzelnen Abstimmungen wie derjenigen zu S21 rund 70\% der Varianz der kommunalen Abstimmungsergebnisse erklären, was auf die herausragende Bedeutung dieser beiden Schlüsselgrössen hinweist.

Grundsätzlich stehen die vorliegenden Resultate in Einklang mit ökonomischen Abstimmungsanalysen, die die geographische Distanz zum Abstimmungsgegenstand bisher im Sinne des traditionellen „Rational Choice“Ansatzes als „self interest“-Indikator verwendet haben (Blöchliger/Spillmann 1992; Schneider/Pommerehne 1985; Schroeder/Sjoquist 1978). So bestätigt sich durchwegs die These des nutzenmaximierenden Stimmbürgers, der je nach örtlicher Nähe zu Bahnlinien, Autostrassen und Theaterbauten zu seinen Gunsten stimmt. Auf eine interessante Ausnahme verweisen hingegen Vatter (1994) sowie Vatter/Nabholz (1995): Zwar bestätigt sich auch bei ihren Abstimmungsstudien zu 20 Infrastrukturvorlagen die hohe Relevanz der Distanzhypothese. Dies trifft jedoch nicht für Volksentscheide zu Schutzbauten vor Natur- und Umweltereignissen (Hochwasser, Lawinen) zu. Offenbar lösen diese Vorlagen eine breite solidarische Unterstützung der Bürgerschaft aus, die das nutzenmaximierende Kalkül der Stimmenden in den Hintergrund rücken lässt. Für die zukünftige Forschung stellt sich deshalb die Frage, ob es weitere Merkmale von Infrastrukturtypen und Abstimmungsprozessen gibt, die zur Überwindung des NIMBY-Verhaltens führen.

29 Diese Befunde bestätigen sich auch auf Individualdatenebene. Vgl. hierzu die VOXUmfragen zu den Schweizer Abstimmungen sowie die Infratest-dimap-Umfragen (Uni Stuttgart) und diejenige der Badischen Zeitung (Uni Freiburg) zu S21. So erweisen sich bei der repräsentativen Bevölkerungsumfrage der Uni Freiburg einzig die Stärke der Parteiidentifikationen mit den Grünen bzw. der CDU als hoch signifikant (Wagschal 2012). 
Die vorliegende Abhandlung hat sich zum Ziel gesetzt, Erkenntnisse zum NIMBY-Stimmbürger am Beispiel der S21-Volksabstimmung und einer Reihe von schweizerischen Volksentscheiden zu liefern. Damit versucht sie auch einen Beitrag zum besseren Verständnis des Stimmverhaltens in Deutschland zu leisten, denn trotz mehr als 2.000 kommunalen Bürgerentscheiden und der im EU-Vergleich größten Zahl von Volksentscheiden auf regionaler Ebene ,wissen wir bisher praktisch nichts darüber, wer diese Verfahren in Deutschland in welcher Weise und aus welchen Gründen nutzt“ (Schoen 2012, S. 514). Unsere empirische Analyse der S21-Volksabstimmung bestätigt dabei einerseits den Hauptbefund von Schoen (2012) zum Stimmverhalten beim Nichtraucherschutz in Bayern von 2010, wonach bei Volksentscheiden in Deutschland referendumsspezifische Merkmale der Bürger (wie in unserem Fall die örtliche Nähe zu S21) eine besonders wichtige Rolle spielen. Gleichzeitig weist sie daraufhin, dass je nach Konfliktmuster und Abstimmungsthema die Parteibindungen der Stimmbürger doch von beträchtlicher Relevanz sein können. Allgemeine Aussagen lassen sich aber erst anhand systematischer Studien von deutschen Volksabstimmungen auf der Basis von Individual- und Aggregatdaten machen, weshalb der Aufforderung von Schoen (2012, S. 534) mit Nachdruck zuzustimmen ist, dass die politikwissenschaftliche Forschung in Deutschland in Zukunft mit ebensolcher Selbstverständlichkeit das Verhalten von Bürgern in direktdemokratischen Prozessen analysieren sollte, wie sie seit Jahrzehnten das Wahlverhalten untersucht. 


\section{Literaturverzeichnis}

Atomschutzverband. 2009. Atom Info: AKW, KKW, Kernkraftwerk Leibstadt. http://www. atomschutzverband.ch/akw-leibstadt.html. Zugriff: 2.11.2011.

Bahnprojekt Stuttgart-Ulm. 2012. 21 gute Gründe für Stuttgart 21. http://pdf.bahnprojekts-ulm.com/21-gute-Gruende-fuer-Stuttgart-21. Zugriff: 13.1.2012.

Baker, Peter J., und D. G. Hoel. 2007. Meta-analysis of standardized incidence and mortality rates of childhood leukaemia in proximity to nuclear facilities. European Journal of Cancer Care 19: 355-363.

Blöchliger, Hansjörg, und Andreas Spillmann. 1992. Wer profitiert vom Umweltschutz? Verteilungswirkungen und Abstimmungsverhalten in Verkehrs- und Umweltvorlagen. Schweizerische Zeitschrift für Volkswirtschaft und Statistik 128: 525-540.

Bolliger, Christian, Yvan Rielle, und Dominik Wyss. 2011. Datendank Swissvotes. http://www.swissvotes.ch. Zugriff: 10.11.2011.

Bowler, Shaun, und Todd Donovan. 1998. Demanding Choices. Opinion, Voting and Direct Democracy. Ann Arbor.

Bühlmann, Marc, Fritz Sager, und Adrian Vatter. 2006. Verteidigungspolitik in der direkten Demokratie. Eine Analyse der sicherheits- und militärpolitischen Abstimmungen in der Schweiz zwischen 1980 und 2005. Zürich: Rüegger.

Bundesamt für Statistik. 2011. Statistisches Lexikon der Schweiz. http://www.bfs.admin.ch/bfs/portal/de/index/infothek/lexikon/lex/0.topic.1.html. Zugriff: 15.11.2011.

Buri, Christof, und Gerald Schneider. 1993. Gründe und Scheingründe für das Schweizer Abstimmungsverhalten. Schweizerische Zeitschrift für Soziologie 19: 389-417.

Dear, Michael. 1992. Understanding and Overcoming the NIMBY Syndrome. Journal of the American Planning Association 58: 288-301.

Dear, Michael, S. Martin Taylor, und G. B. Hall. 1980. External Effects of Mental Health Facilities. Annals of the Association of American Geographers 70: 324-352.

Die Grünen. 2011. Wie geht es jetzt mit Stuttgart 21 weiter? http://gruene-gegenstuttgart21.de/10-gute-grunde-gegen-stuttgart-21/2044. Zugriff: 2.2.2012.

Downs, Anthony. 1957. An Economic Theory of Democracy. New York: Harper and Row.

Ecoplan. 2010. Ersatz-Kraftwerk am Standort Beznau - regionale sozioökonomische Auswirkungen. Bern.

Eder, Christina. 2010a. Direkte Demokratie auf subnationaler Ebene. Eine vergleichende Analyse der unmittelbaren Volksrechte in den deutschen Bundesländern, den Schweizer Kantonen und den US-Bundesstaaten. Baden-Baden: Nomos.

Eder, Christina. 2010b. Ein Schlüssel zum Erfolg? Gibt es ein Patentrezept für Volksentscheide in den deutschen Bundesländern? Politische Vierteljahresschrift 51: 43-67.

Faas, Thorsten, und Schmitt-Beck, Rüdiger. 2012. Tabellenband zur Studie Meinungsbildung, Entscheidfindung und Legitimität politischer Entscheidungen anlässlich der Volksabstimmung zu Stuttgart 21. MZES: Universität Mannheim. 
Freitag, Markus, Adrian Vatter, und Christoph Müller. 2003. Bremse oder Gaspedal? Eine empirische Untersuchung zur Wirkung der direkten Demokratie auf den Steuerstaat. Politische Vierteljahresschrift 44: 348-369.

Freitag, Markus und Uwe Wagschal. 2007. Direkte Demokratie - Bestandsaufnahmen und Wirkungen im internationalen Vergleich. Münster: LIT.

Frey, Bruno S. 1997. Unerwünschte Projekte, Kompensation und Akzeptanz. Analyse und Kritik 19: 3-14.

Frey, Bruno S., Felix Oberholzer-Gee, und Reiner Eichenberger. 1996. The old lady visits your backyard: A tale of morals and markets. Journal of Political Economy 14: 12971312.

Glantz, Alexander, Rebecca Teusch und Harald Schoen. 2011. Abstimmungskampf, Informationsvermittlung und Stimmentscheidung beim Volksentscheid über den Nichtraucherschutz in Bayern. In Jahrbuch für direkte Demokratie 2010. Baden-Baden. Nomos: 295-320.

Greenberg, Michael R., und Samy Amer. 1989. Self-interest and direct legislation: public support of a hazardous waste bond issue in New Jersey. Political Geography Quarterly. 8: 67-78.

Hermann, Michael, und Heiri Leuthold. 2002. Deutsch und Welsch im Raum der Weltanschauungen. Schriftenreihe des Forum Helveticum 10.

Hunter, Susan, and Kevin M. Leyden. 1995. Beyond NIMBY: Explaining Opposition to Hazardous Waste Facilities. Policy Studies Journal 23: 601-619.

Joye, Dominique. 1987. Structure politique et structure sociale: analyse écologique des votations suisses 1982-1980. Grenoble.

Kaatsch, P., C. Spix, S. Schmiedel, R. Schulze-Rath, und M. Blettner. 2006. Epidemiologische Studie zu Kinderkrebs in der Umgebung von Kernkraftwerken (KiKK-Studie). In: Umweltforschungsplan des Bundesumweltministeriums (UFOPLAN). Reaktorsicherheit und Strahlenschutz, Hrsg. Bundesamt für Strahlenschutz. Salzgitter.

Kraft, Michael E., und Bruce B. Clary. 1991. Citizen Participation and the NIMBY Syndrome: Public Response to Radioactive Waste Disposal. Political Research Quarterly 44: 299-328.

Kriesi, Hanspeter. 2005. Direct Democratic Choice. The Swiss Experience. Lanham: Lexington Books.

LeDuc, Lawrence. 2002. Opinion Change and Voting Behavior in Referendums. European Journal of Political Research 41: 711-732.

Linder, Wolf, Regula Zürcher, und Christian Bolliger. 2008. Gespaltene Schweiz - geeinte Schweiz. Gesellschaftliche Spaltungen und Konkordanz bei den Volksabstimmungen seit 1874. Baden: hier + jetzt.

Linder, Wolf, Christian Bolliger, Yvan Rielle. 2010. Handbuch der eidgenössischen Volksabstimmungen 1848 bis 2007. Bern: Haupt.

Lober, Douglas J. 1995. Why Protest? Public Behavioral and Attitudinal Response to Siting a Waste Disposal Facility. Policy Studies Journal 23: 499-518.

Milic, Thomas. 2008. Ideologie und Stimmverhalten. Zürich : Rüegger. 
Nef, Rolf. 1980. Struktur, Kultur und Abstimmungsverhalten. Zur interregionalen Variation von politischen Präferenzen in der Schweiz 1950-1977. Schweizerische Zeitschrift für Soziologie 10: 155-190.

Pommerehne, Werner W. 1975. Öffentliche Güter und öffentliche Aufgaben in der direkten Demokratie. Dissertation, University of Konstanz.

Rabe, Barry G. 1994. Beyond NIMBY: Hazardous Waste Siting in Canada and the United States. Washington, DC: The Brookings Institution.

Rieder, Stefan. 1996. Autobahnbau als Instrument zur Förderung regionaler Wirtschaft? Luzern: Interface Politikstudien.

Schively, Carissa. 2007. Understanding the NIMBY and LULU Phenomena: Reassessing Our Knowledge Base and Informing Future Research. Journal of Planning Literature 21: 255-266.

Schneider, Friedrich, und Werner W. Pommerehne. 1985. A Referendum on a Public Museum's Purchase of Picasso paintings. In Managerial Economics for the Arts, Hrsg. Virginia L. Owen und William S. Hendon, 117-128. Akron.

Schoen, Harald. 2012. Wählen und Abstimmen - Zwei Seiten einer Medaille? Eine Analyse am Beispiel des Volksentscheids zum Nichtraucherschutz in Bayern. In Wählen in Deutschland, Sonderheft Politische Vierteljahresschrift 45: 514-535.

Schroeder Larry D., und Sjoquist David L. 1978. The Rational Voter: An Analysis of Two Atlanta Referenda on Rapid Transit. Public Choice 33: 27-44.

Schwarz, Thomas. 2011. Die Volksabstimmung am 27. November 2011 in Stuttgart. Eine Analyse des Abstimmungsverhaltens in räumlicher und sozialstruktureller Differenzierung. Landeshauptstadt Stuttgart: Statistisches Amt.

Schweizerische Bundeskanzlei. 2011. Chronologie Volksabstimmungen.

Sciarini, Pascal und Anke Tresch. 2009. A two-level analysis of the determinants of direct democratic choices in European, immigration and foreign policy in Switzerland. European Union Politics 10: 456-481.

Stadelmann-Steffen, Isabelle. 2011. Citizens as Veto Players: Climate Change Policy and the Constraints of Direct Democracy. Environmental Politics 20: 485-507.

Statistisches Landesamt Baden-Württemberg. 2011. http:/www.statistik.baden-wuerttemberg.de. Zugriff: 27.1.2012.

Steffen, Isabelle. 2002. Erfolgsfaktoren klimapolitischer Abstimmungsvorlagen. Webdokument, http:// KlimapolitischeAbstimmungen.pdf. Zugriff: 6.12.2011.

Strauf, Simone, Manfred Walser, und Thomas Bieger. 2004. Die wirtschaftlichen Auswirkungen der NEAT-Baustelle in Sedrun auf die Region Obere Surselva.

SVP. 2011a. Der Verkehr. Puls unserer Wirtschaft. http://www.svp.ch/display.cfm/ id/101670. Zugriff: 1.12.2011.

SVP. 2011b. Schweizer Strom. Sicher und umweltfreundlich. http://www.svp.ch/ display.cfm/ id/101658. Zugriff: 1.12.2011.

Van der Horst, Dan. 2007. NYMBY or Not? Exploring the Relevance of Location and the Politics of Voiced Opinions in Renewable Energy Siting Controversies. Energy Policy 35: 2705-2714. 
Vatter, Adrian. 1994. Eigennutz als Grundmaxime in der Politik? Bern, Stuttgart, Wien: Haupt Verlag.

Vatter, Adrian, und Ruth Nabholz. 1995. Der Stimmbürger als Homo Oeconomicus? Ein empirischer Theorientest des Stimmbürgerverhaltens bei kantonalen Kreditvorlagen aus der Schweiz. Politische Vierteljahresschrift 36: 484-501.

Vatter, Adrian, Fritz Sager, Marc Bühlmann und Markus Maibach. 2000. Akzeptanz der schweizerischen Verkehrspolitik bei Volksabstimmungen. NFP41 Schlussbericht. Bern: BBL.

Voll, Peter. 1991. Konfession und Politik in der Schweiz. Zum ,konfessionellen Faktor bei der Entwicklung stabiler Parteisympathien und bei den eidg. Volksabstimmungen vom 9. Juni 1985. Schweizerische Zeitschrift für Soziologie 17: 375-406.

Wagschal, Uwe. 2007. Diskurs oder Machtpolitik? Welche Interessen setzten sich in der Direktdemokratie am erfolgreichsten durch? In Markus Freitag und Uwe Wagschal (Hg.). Direkte Demokratie - Bestandsaufnahmen und Wirkungen im internationalen Vergleich. Münster: LIT: 300-326.

Wagschal, Uwe. 2012. Stuttgart 21 - ein direktdemokratisches Lehrstück? Vortrag gehalten an der Tagung des Konstanzer Wissenschaftsforums. Stein am Rhein. 30.3.2012.

Warren, Charles R., Carolyn Lumsden, Simone O’Down, und Richard V. Birnie. 2005. 'Green on Green': Public Perceptions of Wind Power in Scotland and Ireland. Journal of Environmental Planning and Management 48: 853-875.

Wolsink, Maarten. 1994. Entanglement of Interests and Motives: Assumptions behind the NIMBY-Theory on Facility Siting. Urban Studies 31: 851-867. 


\section{Anhang}

Tabelle 5: Liste der verwendeten Abstimmungen und Resultate

\begin{tabular}{|c|c|c|c|}
\hline $\mathrm{Nr}$. & Datum & Titel der Abstimmungsvorlage & Ja-Stimmen \\
\hline \multicolumn{4}{|c|}{ Stuttgart 21} \\
\hline & 27.11.2011 & S21-Kündigungsgesetz & $41,1 \%{ }^{1}$ \\
\hline
\end{tabular}

Bahnabstimmungen

Nr. $348 \quad 6.12 .1987$ Bundesbeschluss betreffend das Konzept Bahn 57,0\% 2000

Nr. 382 27.9.1992 Bundesbeschluss über den Bau der schweizeri- $63,6 \%$ schen Eisenbahn-Alpentransversalen (Alpentransit-Beschluss)

Nr. 445 29.11.1998 Bundesbeschluss über Bau und Finanzierung 63,5\% von Infrastrukturvorhaben des öffentlichen Verkehrs

Autobahnabstimmungen

\begin{tabular}{|c|c|c|}
\hline Nr. 359 & 1.4 .1990 & Volksinitiative „Stopp dem Beton“ \\
\hline Nr. 360 & 1.4 .1990 & $\begin{array}{l}\text { Volksinitiative "für eine autobahnfreie Land- } \\
\text { schaft zwischen Murten und Yverdon“ }\end{array}$ \\
\hline Nr. 361 & 1.4 .1990 & $\begin{array}{l}\text { Volksinitiative „für ein autobahnfreies Knonau- } \\
\text { er Amt“ }\end{array}$ \\
\hline
\end{tabular}

Nr. 362 1.4.1990 Volksinitiative „für eine autobahnfreie Aare- 34,0\% ${ }^{1}$ landschaft zwischen Biel und Solothurn/Zuchwil“"

Nr. 504 8.2.2004 Gegenentwurf der Bundesversammlung zur $\quad 37,2 \%$

Volksinitiative „Avanti - für sichere und leis-

tungsfähige Autobahnen“"

Atomkraftwerkabstimmungen

Nr. 321 23.9.1984 Volksinitiative ,für eine Zukunft ohne weitere 45,0\% Atomkraftwerke“

Nr. 365 23.9.1990 Volksinitiative „Stopp dem Atomkraftwerkbau 54,5\% (Moratorium)“

Nr. 366 23.9.1990 Volksinitiative ,für den Ausstieg aus der Atom- 47,1\% ${ }^{1}$ energie“"

Nr. $501 \quad$ 18.05.2003 Volksinitiative „MoratoriumPlus“ $\quad 41,6 \%{ }^{1}$

Nr. $502 \quad 18.05 .2003$ Volksinitiative ,Strom ohne Atom“ $33,7 \%{ }^{1}$

${ }^{1}$ Die Ergebnisse dieser Vorlagen mussten für die Analyse ,gedreht“" werden, da sie in ihrer ursprünglichen Form eine Verhinderung von Infrastrukturprojekten vorsahen. 
Tabelle 6: Liste der verwendeten Variablen

\section{Stuttgart 21:}

\begin{tabular}{llll} 
AV: & Ja-Stimmen-Anteil & $\begin{array}{l}\text { Prozentualer Ja-Stimmen- } \\
\text { Anteil in den Gemeinden }\end{array}$ & Stat. Landesamt BW \\
UV: & Distanz & $\begin{array}{l}\text { Distanz in km } \\
\text { Beteiligungsquote in den }\end{array}$ & $\begin{array}{l}\text { Stat. Landesamt BW } \\
\text { Stat. Landesamt BW } \\
\text { Gemeinden (in \%) }\end{array}$ \\
& Stärke der Grünen & $\begin{array}{l}\text { Stärke der Grünen Partei in } \\
\text { den Gemeinden (bei den }\end{array}$ & Stat. Landesamt BW \\
& $\begin{array}{l}\text { Landtagswahlen 2011) } \\
\text { Bevölkerungsgröße der }\end{array}$ & Stat. Landesamt BW \\
Bevölkerungsgröße & $\begin{array}{l}\text { Stimmkreise, gemittelt } \\
\text { Urbanisierungsgrad der }\end{array}$ & Stat. Landesamt BW \\
& Urbanisierungsgrad & $\begin{array}{l}\text { Stimmkreise, gemittelt } \\
\text { Wirtschaftliche Leistungs- }\end{array}$ & Stat. Landesamt BW \\
kirtschaftliche Leis- & $\begin{array}{l}\text { kraft der Stimmkreise, ge- } \\
\text { mittelt }\end{array}$ & \\
\hline & &
\end{tabular}

Schweizer Abstimmungen:

\begin{tabular}{|c|c|c|c|}
\hline AV: & Ja-Stimmen-Anteil & $\begin{array}{l}\text { Prozentualer Ja-Stimmen- } \\
\text { Anteil in den Gemeinden }\end{array}$ & Bundeskanzlei 2011 \\
\hline \multirow[t]{11}{*}{ UV: } & Distanz & Distanz in $\mathrm{km}$ & www.luftlinie.org \\
\hline & Abst. Nr. & Abstimmungsnummern & Bundeskanzlei 2011 \\
\hline & Beteiligung & $\begin{array}{l}\text { Beteiligungsquote in den } \\
\text { Gemeinden (in \%), gemittelt } \\
\text { für die Kantone }\end{array}$ & Bundeskanzlei 2011 \\
\hline & Koalitionen & $\begin{array}{l}\text { Koalitionen auf Kantons- } \\
\text { ebene für Vorlage }\end{array}$ & Bolliger 2011 \\
\hline & Stärke der GPS & $\begin{array}{l}\text { Stärke der Grünen Partei in } \\
\text { den Gemeinden (bei den } \\
\text { letzten NR-Wahlen) }\end{array}$ & $\begin{array}{l}\text { Bundesamt für Sta- } \\
\text { tistik (BfS) } 2011\end{array}$ \\
\hline & Stärke der SVP & $\begin{array}{l}\text { Stärke der SVP in den Ge- } \\
\text { meinden (bei den letzten } \\
\text { NR-Wahlen) }\end{array}$ & BfS 2011 \\
\hline & Sprachregion & Sprachregion & BfS 2011 \\
\hline & Religion & $\begin{array}{l}\text { Prozentualer Anteil Katholi- } \\
\text { ken }\end{array}$ & BfS 2011 \\
\hline & Bevölkerungsgrösse & $\begin{array}{l}\text { Kantonale Bevölkerungs- } \\
\text { grösse, gemittelt }\end{array}$ & BfS 2011 \\
\hline & Urbanisierungsgrad & $\begin{array}{l}\text { Kantonaler Urbanisierungs- } \\
\text { grad, gemittelt }\end{array}$ & BfS 2011 \\
\hline & $\begin{array}{l}\text { Wirtschaftliche Leis- } \\
\text { tungskraft }\end{array}$ & $\begin{array}{l}\text { Kantonale wirtschaftliche } \\
\text { Leistungskraft, gemittelt }\end{array}$ & BfS 2011 \\
\hline
\end{tabular}


Abb. 1.1 und 1.2: Marginale Effekte der S21-Abstimmungen, Gemeindeebene
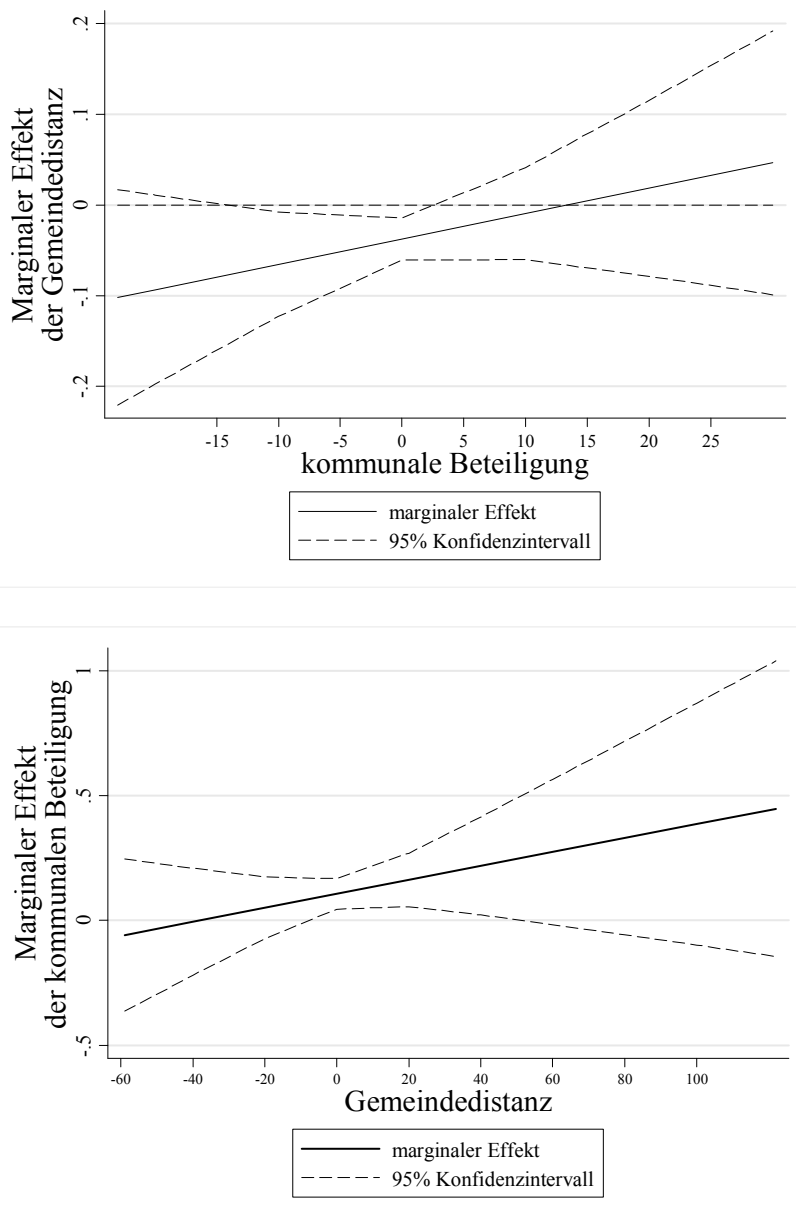
Abb. 1.3 und 1.4: Marginale Effekte der S21-Abstimmungen, Kreisebene
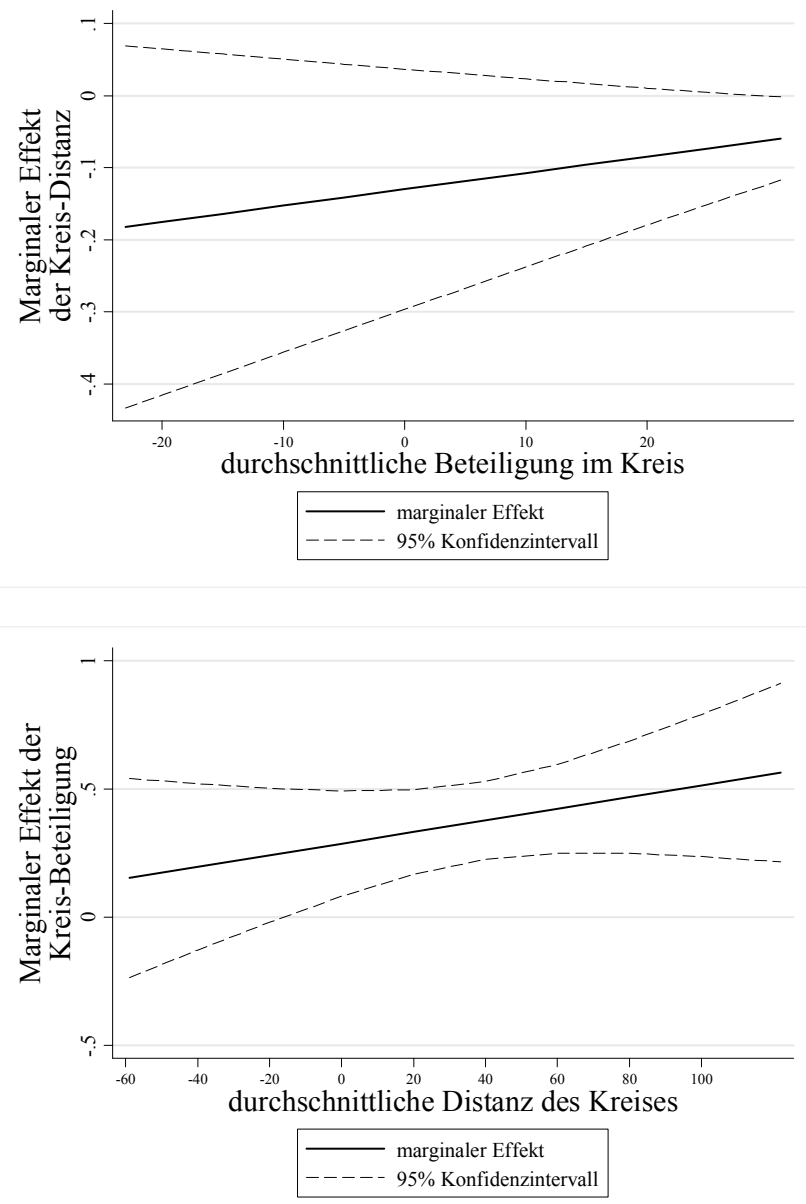
Abb. 2.1 und 2.2: Marginale Effekte der Bahn-Abstimmungen, Gemeindeebene
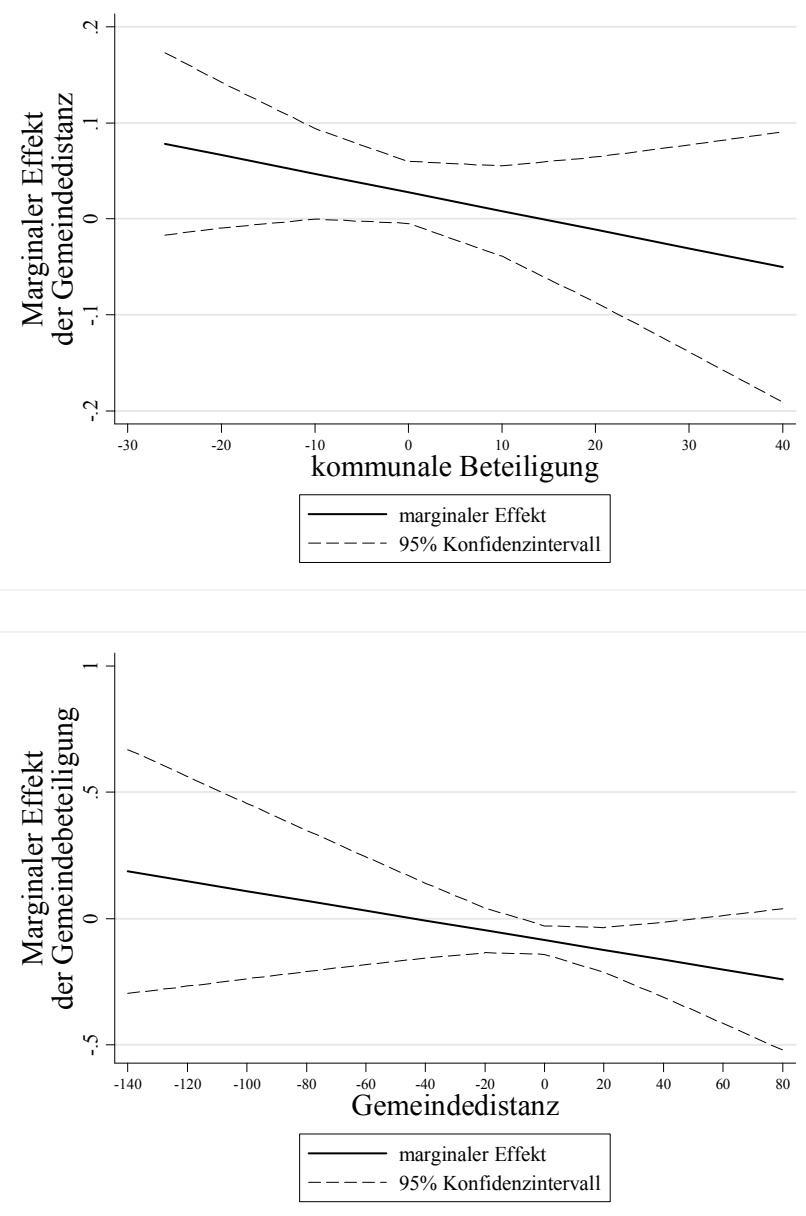
Abb. 2.3 u. 2.4: Marginale Effekte der Bahn-Abstimmungen, Kantonsebene
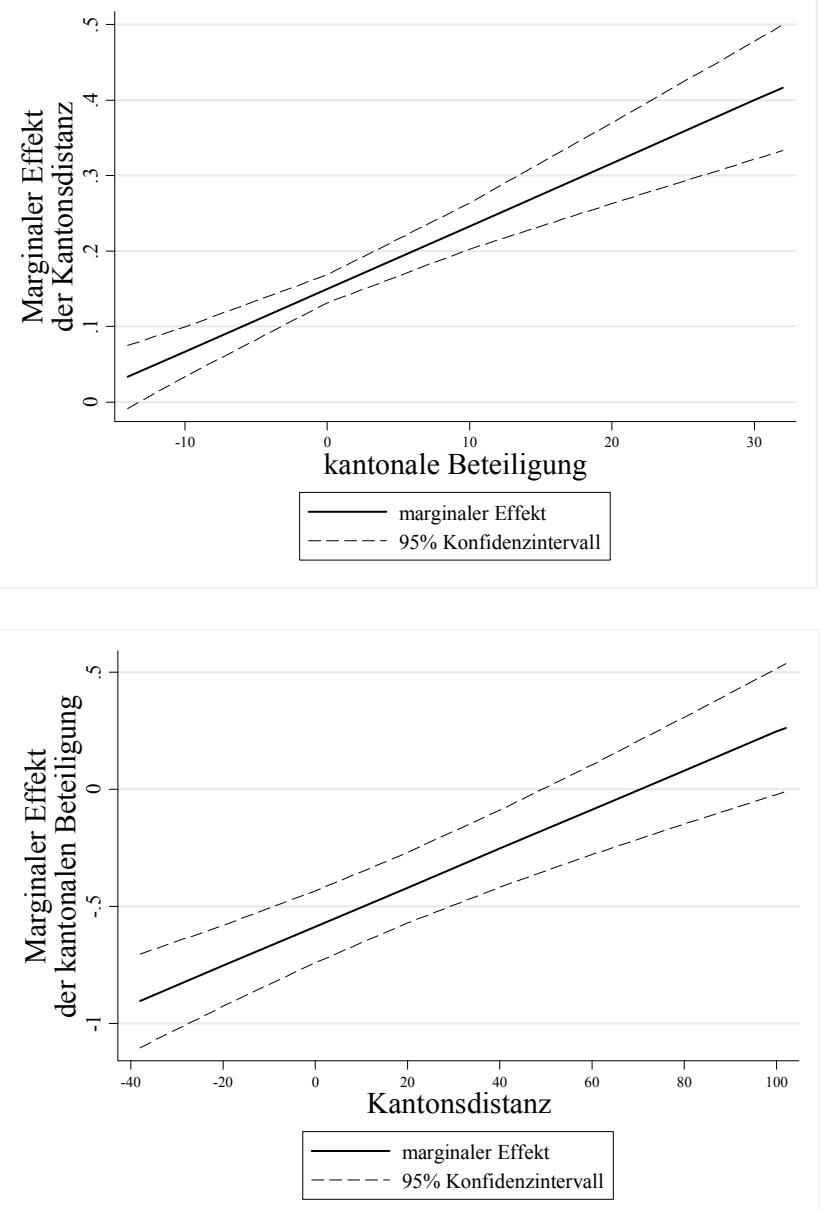
Abb. 3.1 und 3.2: Marginale Effekte der Autobahn-Abstimmungen, Gemeindeebene
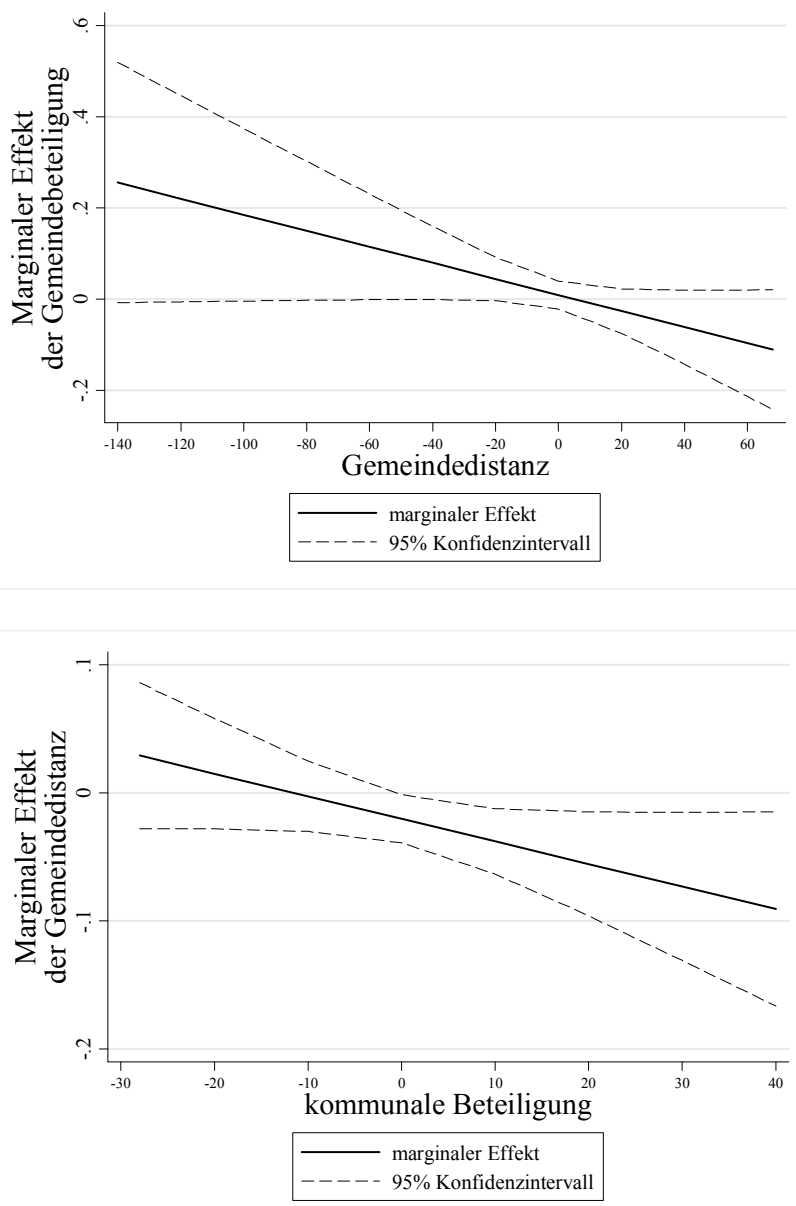
Abb. 3.3 und 3.4: Marginale Effekte der Autobahn-Abstimmungen, Kantonsebene
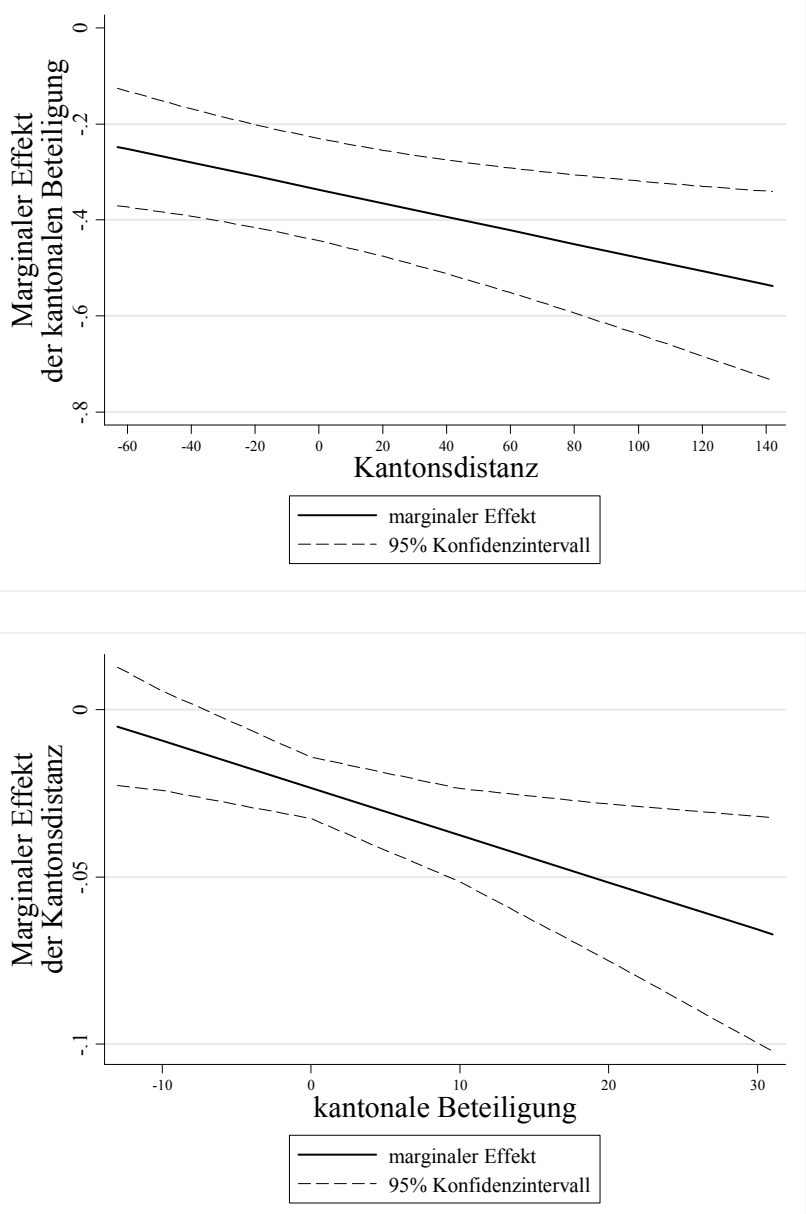
Abb. 4.1 und 4.2: Marginale Effekte der AKW-Abstimmungen, Gemeindeebene
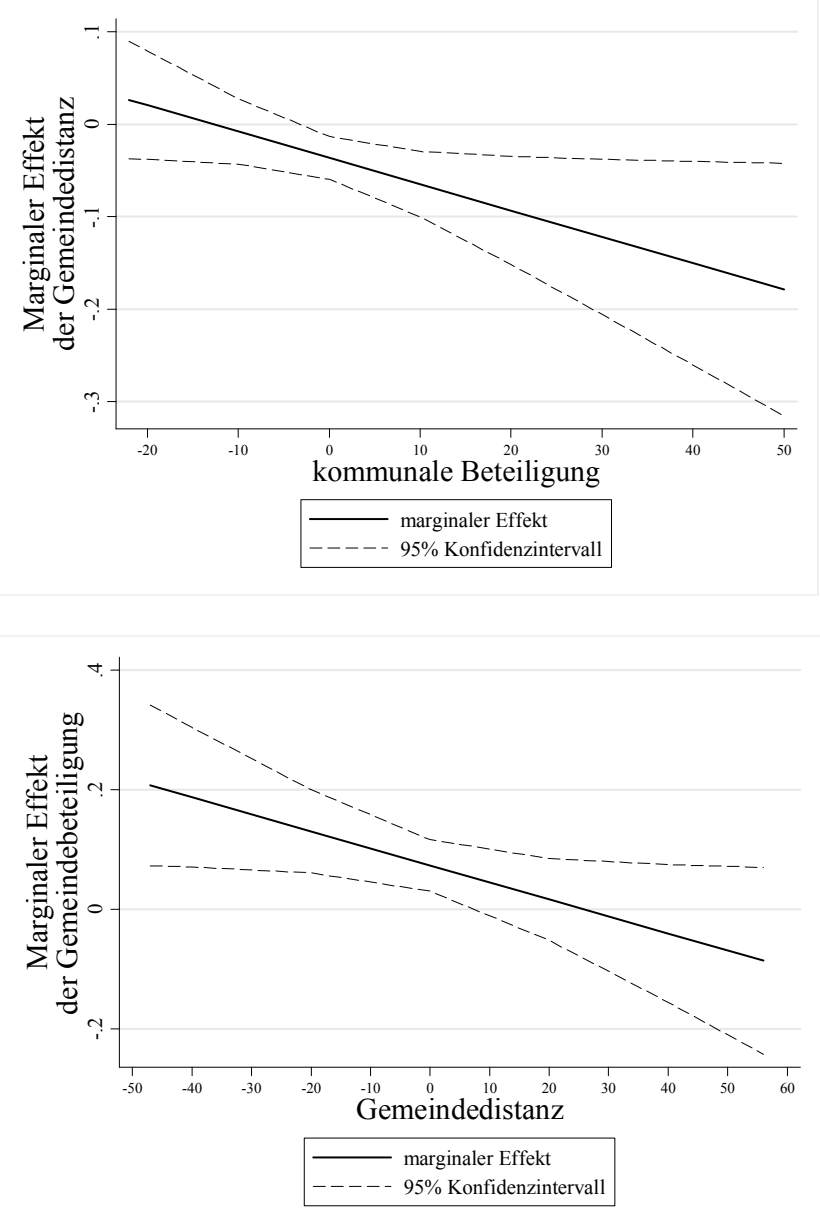
Abb. 4.3 u. 4.4: Marginale Effekte der AKW-Abstimmungen, Kantonsebene
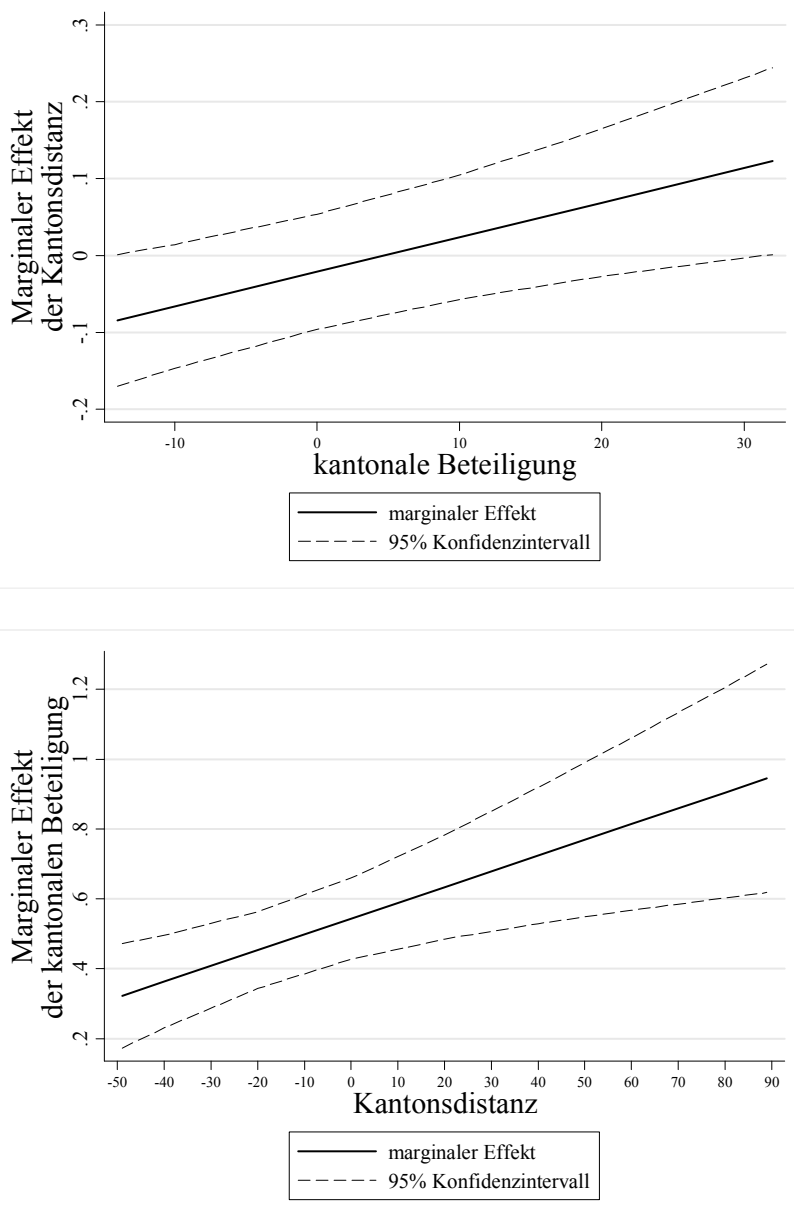